Accepted refereed manuscript of:

Cornwall M \& Hagermann A (2016) Planetary heat flow from shallow subsurface measurements: Mars, Planetary and Space Science, 131, pp. 46-59.

DOI: $\underline{10.1016 / \text { i.pss.2016.07.001 }}$

(C) 2016, Elsevier. Licensed under the Creative Commons AttributionNonCommercial-NoDerivatives 4.0 International http://creativecommons.org/licenses/by-nc-nd/4.0/ 


\title{
Planetary Heat Flow from Shallow Subsurface Measurements: Mars
}

\author{
Marc Cornwall ${ }^{1}$, Axel Hagermann \\ Department of Physical Sciences, The Open University, Walton Hall, Milton Keynes, MK7 6AA, United Kingdom \\ ${ }^{1}$ Email: marc.cornwall@gmail.com | Phone: +44(0)7968 415355 | Fax: +44(0)1908 655667
}

Keywords: mars | heat flow | inversion | geophysics

\section{Abstract}

Planetary heat flow probes measure heat flow (depth-resolved temperature and thermal conductivity) to provide insight into the internal state of a planet. The probes have been utilized extensively on Earth, twice on the Moon, and once on the Surface of comet 67P-CG. Mars is an important target for heat flow measurement as heat flow is a critical parameter in Martian thermal history models. Earlier studies indicate that Martian planetary heat flow can be accessed at $5 \mathrm{~m}$ below the surface in dry regolith monitored over at least one Martian year. A one Martian year monitoring period is necessary because, in the shallow subsurface, heat flow from the interior is superposed with time varying heat flow contributions, primarily due to insolation. Given that a heat flow probe may not achieve its target depth or monitoring period, this study investigates how the depth (2-5 m), duration (0-1 Martian year) and quality of measurements influence the accuracy of planetary heat flow. An inverse model is used to show that, in the preceding scenarios, the accuracy of planetary heat flow directly estimated from depth-dependent thermal conductivity with 10-20 \% precision errors, temperatures with 50-100 mK precision errors and modelling uncertainties up to $500 \mathrm{mK}$, can, on average, be improved by a factor of 27 with optimization to $13 \%$. Accuracies increase with sensor penetration depth and regolith monitoring period. Heat flow optimized from instantaneous measurements or those with the shortest regolith monitoring periods have increased accuracy where the frequency and amplitude of the temperature variation are lowest. The 
inverse model is based on the Function Specification Inversion method. This study demonstrates that a solution subspace can be identified within a space of uncertainties modelled for the temperature measurements and planetary heat flow: the subspace is defined by a constant log-ratio of their respective standard deviations. Optimized heat flow estimates display reduced correlation with increasing temperature precision and systematic conductivity errors, with the constraint of other known model parameters. Consequently, the model permits upper bounds to be placed on the conductivity estimate without conductivity optimization, as heat flows are optimized to a limiting value with increasing systematic conductivity errors for any given parameter set. Overall, the results demonstrate a $52 \%$ chance of achieving a direct heat flow estimate accurate to within $40 \%$, with the same being $82 \%$ after optimization.

\section{Introduction}

\subsection{Planetary Heat Flow}

Planetary heat flow quantifies heat flow from the interior of a planet and sheds light on related interior processes (e.g. Hagermann, 2005): it is the product of the thermal conductivity and background (mean) subsurface temperature gradient and can therefore be obtained, to first order, by performing depth-resolved measurements of conductivity and temperature. In a planet's shallow subsurface, planetary heat flow is superimposed with heat flow due to insolation, which is usually several orders of magnitude greater. However, heat flow from insolation is exponentially damped with depth; therefore, planetary heat flow becomes more isolated at depth. The skin depth, over which the amplitude of the heat flow

due to insolation drops by a factor of $e$, quantifies the former. If the depth of measurement is not sufficiently far below the skin depth, or the duration of measurement too short to fully sample the temperature variation, advanced methods to isolate the planetary heat flow are required (e.g. Spohn et al., 2001; Grott et al., 2007). 


\subsection{Measuring Martian Heat Flow}

Grott et al. (2007) model Martian skin depths of 0.65 and $1.45 \mathrm{~m}$. They estimate that a heat flow probe (a linear suite of temperature and conductivity sensors emplaced to a given depth - e.g. Spohn et al., 2001; Hagermann, 2005) can measure an associated Martian heat flow of $20 \mathrm{~mW} / \mathrm{m}^{2}$ to an accuracy within $30 \%$, given measurements over at least a Martian year, depths of at least 3 or $5 \mathrm{~m}$ corresponding to each skin depth, temperature measurement precision of $0.1 \mathrm{~K}$ and conductivities at least $20 \%$ accurate. Dehant et al. (2012) demand a higher temperature precision of $0.05 \mathrm{~K}$ for depths up to $2 \mathrm{~m}$.

Heat flow probes have been used to take heat flow measurements thousands of times on Earth (e.g. Pollack et al., 1993) and twice on the Moon (e.g. Langseth et al., 1972; 1976; Heiken et al., 1991). The measurements have provided insights into the radioisotope distribution within the crusts of the Earth (e.g. Rudnick and Fountain, 1995; Boehler, 1996) and the Moon (e.g. Warren and Rasmussen, 1987; Hagermann and Tanaka, 2006). Similar insights are possible with a heat flow measurement on Mars (e.g. Dehant et al., 2012). Heat Flow and Physical Properties Package $\left(\mathrm{HP}^{3}\right)$ is a proposed heat flow probe on NASA's InSight lander, which satisfies the measurement depth, time and precision requirements outlined at the beginning of this section (Banerdt et al., 2012; Spohn et al., 2012; Golombek et al., 2014; NASA, 2014).

If a measurement falls short of the requirements, inverse modelling of critical parameters can potentially recover the Martian heat flow; this has been used to similar effect on Earth, though over km depth scales (e.g. Shen and Beck, 1991; 1992; Wang, 1992), avoiding shallow depth effects of the temperature variation. Mass and cost restrictions limit the current generation of planetary probes to shallow-depth measurements. Here, a numerical inversion algorithm is tested against the potential measurement shortfalls that can occur at shallow depth with a heat flow probe measurement on Mars. 


\subsection{Experimental Procedure}

The investigation proceeds as follows: in Section 2, a theoretical model of the Martian heat flow environment is presented (Section 2.1) with a procedure for inversion of a temperature profile for heat flow, and potentially other critical parameters (Sections $2.2-2.3$. Errors associated with the models are discussed in Section 2.4; in Section 3, critical parameters of the heat flow model are discussed in the context of the Martian environment; in Section 4, example simulations with the heat flow model parameters are presented and discussed with associated synthetic measurement scenarios. Results of applying the inversion procedure to the synthetic measurements are presented in Section 5 and discussed in Section 6.

\section{Model of Martian Thermal Environment}

\subsection{Heat Flow Equation}

The flux of heat through the surface of a planet can be expressed using the one-dimensional heat flow equation. For the current purposes, it is most useful to partition the equation into two components: the first due to the surface temperature variation, such that

$$
\rho c(z) \frac{\partial T^{U}(z, t)}{\partial t}-\frac{\partial}{\partial z}\left(k(z) \frac{\partial T^{U}(z, t)}{\partial z}\right)=S^{U}(z, t)=0, z \in\left[z_{S}, z_{B}\right], t \in i,
$$

with boundary and initial conditions

a.

$$
T^{U}(z, t)=T_{S}^{U}(t), z=z_{S}, t \in i,
$$

b.

$$
-k(z) \frac{\partial T^{U}(z, t)}{\partial z}=F_{B}^{U}(z, t)=0, z \in\left[z_{S}, z_{B}\right], t \in i,
$$

c.

$$
T^{U}(z, t)=T^{U 0}(z)=0, z \in\left[z_{S}, z_{B}\right], t=t_{B} ;
$$

and the second due to the planetary heat flow, such that

$$
\frac{-\partial}{\partial z}\left(k(z) \frac{\partial T^{S}(z)}{\partial z}\right)=S^{S}(z), z \in\left[z_{S}, z_{B}\right]
$$


with boundary conditions

$$
T^{S}(z)=T_{S}^{S}, z=z_{S}
$$

b. $-k(z) \frac{\partial T^{S}(z)}{\partial z}=F_{B}^{S}, z=z_{B}$,

where ${ }^{t}$ is time when the monitoring period begins $\left({ }^{t}\right)$ and stops $\left({ }^{t} s\right) ;{ }^{z}$ is depth at the surface $\left({ }^{z_{s}}\right)$ and base $\left({ }^{z_{B}}\right)$ of the regolith section; $\rho c(z)$ is the thermal capacity, the product of the depth dependent density $\rho(z)$ and specific heat (at constant pressure) ${ }^{c}$; and $k(z)$ is the thermal conductivity. $S^{U}(z, t)$ and $S^{S}(z)$ are terms representing heat sources and sinks, where $S^{U}(z, t)$, while conventionally set to 0 , is expressed here as it is an important parameter in FSI inversion (it can also be used to reproduce the effects of temperature dependent thermal properties). The temperature variation $T^{U}(z, t)$ and the background (mean) temperature due to the heat flow $T^{S}(z)$ sum to the physical temperature $T(z, t)$. The boundary conditions reflect a temperature variation entirely due to the surface temperature variation $T_{S}^{U}(t)$ (Equation a) while $T_{S}^{S}$ (Equation a) represents the background (mean) surface temperature. The lower boundary condition of the background temperature equation (Equation b) is the planetary heat flow $F_{B}^{S}$. Equations - can be solved either analytically or numerically to give the physical temperature $T(z, t)$. 


\subsection{Inverse Theory}

To determine heat flow, measurements of the temperature and conductivity profiles must be made as noted in Section 1.1. The measurements will contain noise (Section 2.4), which decreases the accuracy to which planetary heat flow can be directly determined. The accuracy of planetary heat flow, directly measured in the former manner, can be improved by inverting the temperature measurement for the planetary heat flow $F_{B}^{S}$ and other unkown parameters.

The temperature measurement can be represented

by $d \Leftrightarrow T^{U}(z, t)+T^{S}(z)+\eta(z, t)=T(z, t)+\eta(z, t) ; T(z, t)$ being the pristine temperature (solution of Equations -) and $\eta(z, t)$ being the noise associated with a temperature measurement, such that

$$
d=g(m)
$$

Variable $m=\left[T_{S}^{U}, T_{S}^{S}, F_{B}^{S}, k, \rho, c, S\right]$ is a vector of thermal model parameters where $g(m)$ is the constraining model. Because $g(m)$ may be largely unknown and $d$ is noisy, Equation represents a sparse linear system and can be solved using relevant methods.

In this investigation a Bayesian least squares functional space inversion method (e.g. Tarantola and Valette, 1982, Shen and Beck, 1991,1992; Tarantola, 2005) is used to invert the temperature measurements. The critical procedure with this method is the optimization of a misfit function $\Sigma$, defined as

$$
\sum=\frac{1}{2}\left[\left(g(m)-d_{0}\right)^{T} C_{d}^{-1}\left(g(m)-d_{0}\right)+\left(m-m_{0}\right)^{T} C_{m}^{-1}\left(m-m_{0}\right)\right] .
$$


Variable $m_{0}$ is a vector of initial parameter estimates (measurements) and $d_{0}$ is a vector of temperature measurements. $C_{d}$ and $C_{m}$ are covariance operators which respectively quantify the uncertainties in the temperature measurements and initial thermal model parameter estimates. $\Sigma$ is optimized using the quasi-Newton method of steepest descent (e.g. Tarantola, 2005) where ${ }^{m}$ is updated from the gradient $Y$ of $\sum$ according to (Shen and Beck, 1991)

$$
m_{i+1}=m_{i}-\mu_{i} \gamma_{i}=m_{i}-\mu_{i}\left(\frac{\partial \sum}{\partial m}\right)_{i} .
$$

The calculation of gradient $Y$ is summarised in Appendix $\mathrm{A}$.

\subsection{Misfit Function Optimization}

For the quasi-Newton descent optimization method, the stepping factor $\mu \approx_{1}$, with its precise value dependent on the geometry of the problem. The method, in principle, allows for simultaneous optimization of all components of ${ }^{m}$ (see Shen and Beck, 1992), though here the focus is on optimization of $F_{B}^{S}$, the planetary heat flow, therefore $m=\left[F_{B}^{S}\right]$. The procedure starts from an initial estimate of Martian heat flow such that $m_{0}=\left[F_{B}^{S 0}\right]$ which can be obtained from, for example, the temperature and thermal conductivity measurements. It proceeds iteratively, ideally with the respective minimisation and maximization of $L_{2}$ norms $\left\|g(m)-d_{0}\right\|$ and $\left\|m-m_{0}\right\|$ in Equation, with the consequent optimization of misfit function $\Sigma$. 
The iterative path (convergence) of $\Sigma$ is dependent on the magnitudes of the standard deviations within $C_{m}$ and $C_{d}$, which may be unknown to some degree. If planetary heat flow standard deviation $\sigma_{F_{B}^{s} \text { in }} C_{m}$ is set too small relative to temperature measurement standard deviation $\sigma_{d \text { in }} C_{d}$, the minimum of $\sum$ may not correspond to the maximum of $\left\|m-m_{0}\right\|$ which contains the most optimal model in ${ }^{m}$ (Figure 1a). To mitigate this uncertainty, $C_{m}$ and $C_{d}$ can be used to define a two-dimensional, finite problem space with optimal points of $\sum$, a subspace of which contains the required solutions to the inverse problem (Figure 1b). The solution subspace is well defined for planetary heat flow $F_{B}^{S}-$ it is a region where the logratio of $\sigma_{F_{B}^{s}}$ to $\sigma_{d}$ is at least equal to a constant $r_{d}^{F_{B}^{s}}$, here called the stability ratio, such that

$$
\log _{10} \sigma_{F_{B}^{s}} \geq r_{d}^{F_{B}^{S}} \log _{10} \sigma_{d}+b, b \in R^{i}
$$

The stability ratio for a given problem can be discerned by inverting the given parameter set over a range of $\sigma_{F_{B}^{s}}$ and $\sigma_{d}$, and plotting the resulting values of $\Sigma$ in the problem space. As illustrated in Figure $1 \mathrm{~b}$, the former should clearly reveal a linear region, intersecting the ${ }_{F_{\mathrm{B}^{-}}^{s}}$ $\sigma_{d}$ plane, thus identifying $r_{d}^{F_{B}^{s}}$ and the solution space (given the parameter set is physically meaningful and/or close enough to the true values). The distinct form of $\sum$ in the $\sigma_{F_{B}^{s}} \sigma_{d}$ space is analogous to a potential barrier (Figure 1c) located at the line defined by Equation , 
where the gradients of $\left\|g(m)-d_{0}\right\|$ and $\left\|m-m_{0}\right\|$ respectively achieve maxima and minima, with respect to ${ }_{F_{B}^{s}}$. The most viable solutions are located beyond the potential barrier in the more stable region of the $\sigma_{F_{B}^{s}} \sigma_{d}$ space. The planetary heat flow estimates $F_{B}^{S}$ can be plotted instead of $\sum$, which should reveal a distinct dichotomy, marked (intersected) by the $r_{d}^{F_{B}^{s}}$ line, where, for $\sigma_{F_{B}} \rightarrow 0, F_{B}^{S}$ is close to $F_{B}^{S 0}$ and for $\sigma_{F_{B}^{S}}{ }_{\infty}, F_{B}^{S}$ is close to the optimal heat flow value for the given parameter set (Figure $1 \mathrm{~d}$ ). The stability ratio $r_{d}^{F_{B}^{s}}$ is equivalent to the gradient of the line, calculated from the logarithm of the standard deviations.
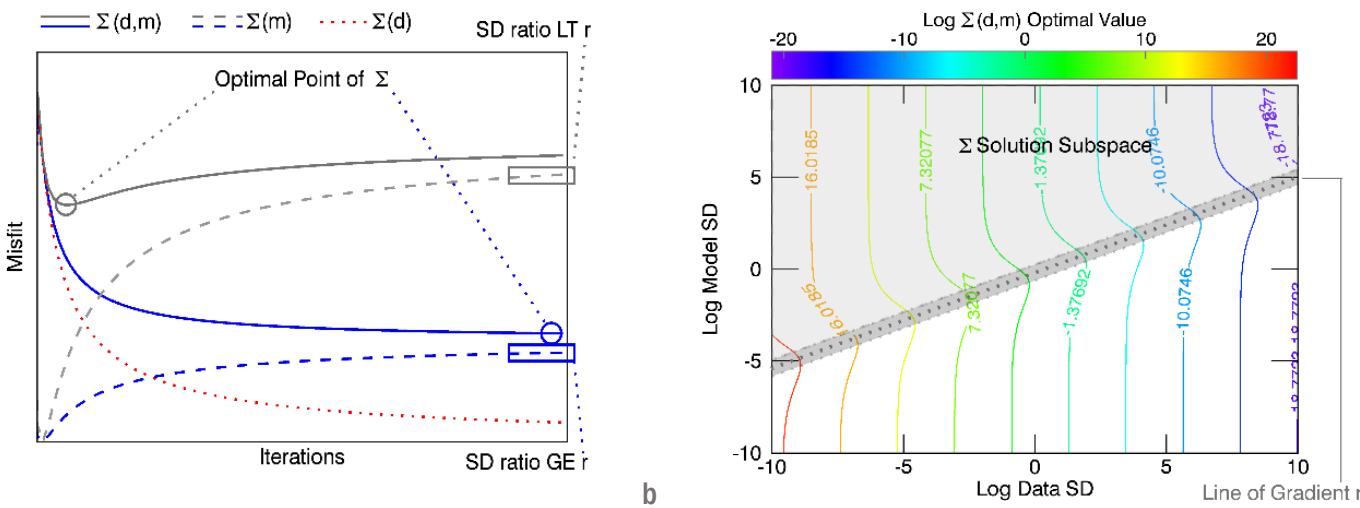

a
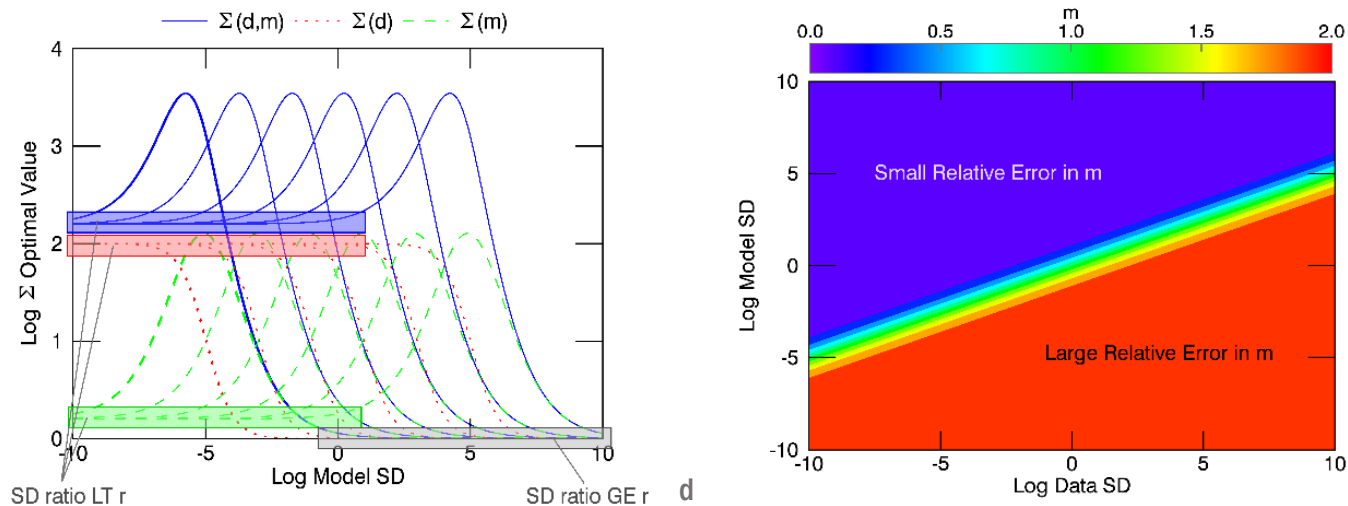

Figure 1. Properties of misfit function $\sum$ and solution space of optimal $\sum$ values (idealised): a. iterative path (convergence) of

$\sum$ (solid curves) with the dashed curves being the maximised model parameter misfit $\sum(m)=\left\|m-m_{0}\right\|$ and the dotted 
curve the minimised data misfit $\sum(d)=\left\|g(m)-d_{0}\right\| ;$ b. form of $\sum$ in the $\sigma_{F_{B^{-}}^{s}} \sigma_{d}$ space. Note the significant decrease in

$\sum$ due only to the increasing values of $\sigma_{d}$ (this effect is normalised in plot c); c. form of $\sum(d, m)$ and it's components

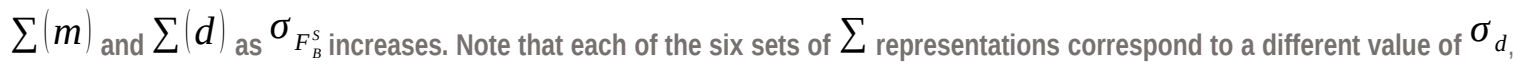

with the value of $\sum$ normalised to the effects of increasing $\sigma_{d}$ seen in b; d. form of the optimal values of $m$ plotted in the $\sigma_{F_{B^{-}}^{s}}$ $\sigma_{d}$ space such that the solution subspace can be identified immediately from a distribution of $F_{B}^{S}$ values.

\subsection{Model Errors}

The inverse simulations can be run from an initial background heat flow estimate $\left(F_{B}^{S 0}\right)$ calculated directly from the mean heat flow through the two lowest sensors (Figure 4) in each measurement. Equations $b$ and $b$ allow a theoretical estimate of the error in a directly calculated heat flow $F$, given errors in conductivity, temperature and/or depth according to

$$
\frac{\Delta F}{F}=\sqrt{\left(\frac{\Delta k}{k}\right)^{2}+\left(\frac{\Delta d T}{d T}\right)^{2}+\left(\frac{\Delta d z}{d z}\right)^{2}}
$$

where $d T(0.02-0.1 \mathrm{~K})$ and $d z(23 \mathrm{~cm})$ account for temperature and depth differences between the uppermost and lowermost sensor used in the calculation. There are two ways of calculating the background heat flow: 1 . the mean of a series of temperatures measured at $m$ discrete times can be found at each depth over the entirety of the monitoring period,

and then used to calculate a mean heat flow such that $\Delta d T=T \frac{\sqrt{2 m} \Delta T_{m}}{\sum_{m} T_{m}}$ or; $2 .{ }^{m}$ discrete heat flows can be calculated at each sensor depth, then averaged over the monitoring 
period to give the mean heat flow such that $\Delta F=F \frac{\sqrt{m} \Delta F_{m}}{\sum_{m} F_{m}}$ where $\Delta F_{m}$ is given by Equation .

Given accurate and precise sensor location data $(\Delta z=0)$, temperature measurement errors $\Delta T$ of $50 \mathrm{mK}$ (precision) - $500 \mathrm{mK}$ (modelling), conductivity measurement errors of $10-20 \%$ and pristine heat flows of $5-25 \mathrm{~mW} / \mathrm{m}^{2}$, the calculated heat flows are typically $15-35 \%$ accurate where $669^{\geq m} \geq_{167}$ temperature profiles ( 1 per Sol) are averaged to get the background temperature, then used to calculate a heat flow (method 1 above). However, the calculated heat flows are inaccurate by factors of 0.06 (for $25 \mathrm{~mW} / \mathrm{m}^{2}$ pristine heat flow) to 8 where the corresponding heat flow profiles are calculated, then averaged to get background heat flow (method 2 above). For single temperature profiles where ${ }^{m=} 1$ (an instantaneous measurement), the inaccuracies are the same for both methods. Method 1 is more sensitive to conductivity errors than to temperature errors (which are averaged); the converse holds true for method 2 .

The precise results of optimization using the steepest descent method outlined in Sections 2.2-2.3 display marginal dependence on the initial heat flow estimate; therefore, optimization is carried out with heat flows calculated from both methods ( 1 and 2 above) as different starting points for each measurement scenario.

\section{Martian Heat Flow Parameters}

\subsection{Measurement Locations}

The choice of heat flow measurement location is critically important. Generally, locations are ideally dry, to avoid transient perturbation of thermal properties by ices (e.g. Grott et al., 
2007), flat and homogeneous, to avoid perturbations of the temperature gradient by surface and subsurface geology (e.g. Wang, 1992; Golombek et al. (2014). For the purposes herein, measurements are synthesised from properties and timings of the proposed InSight landing ellipses (within $134^{\circ} \mathrm{E} 3^{\circ} \mathrm{N}$ and $139.5^{\circ} \mathrm{E} 5^{\circ} \mathrm{N}$ around Ls 252.7 ; Golombek et al., 2014) in the Elysium region, which is relatively flat (e.g. Golombek et al., 2013; Wigton et al., 2014), with a fine regolith layer (e.g. Ruff and Christensen, 2002; Nowicki and Christensen, 2007; Warner et al., 2014) typically $10 \mathrm{~m}$ thick (Golombek et al., 2013). The equatorial location reduces the chance of encountering ices in the regolith (e.g Boynton et al., 2002).

\subsection{Martian Heat Flow}

Different thermal models use combinations of so-called plate cooling models (involving plate tectonics; e.g. Breuer and Spohn, 2003) and/or stagnant lid models (involving a one-plate lithosphere; e.g. Hauck and Phillips, 2002) to nominally estimate current Martian global heat flow in the range $5-25 \mathrm{~mW} / \mathrm{m}^{2}$ (e.g. Dehant et al., 2012). Mars is currently thought to be in the stagnant lid regime with heat flow from the selected region in Section 3.1 estimated to be of the order of $15-20 \mathrm{~mW} / \mathrm{m}^{2}$ (Grott and Breuer, 2010). For comparison purposes, measurements from the region are synthesised with end-member heat flows of 5 and 25 $\mathrm{mW} / \mathrm{m}^{2}$.

\subsection{Surface Temperature Mean and Variation}

The Martian surface temperature can be modelled from large scale simulations such as Mars Climate Database (MCD - Millour et al., 2012) and Mars Global Reference Atmospheric Model (Mars-GRAM - Justh et al., 2011) which integrate climate data from the many orbiters, landers and rovers which continually study the planet. Figure 2 shows MarsGRAM surface temperature for a measurement site within the region identified in Section 3.1; the temperature varies diurnally (light-toned red curves) over $90 \mathrm{~K}$ and seasonally (midtoned red curves) over $30 \mathrm{~K}$. 


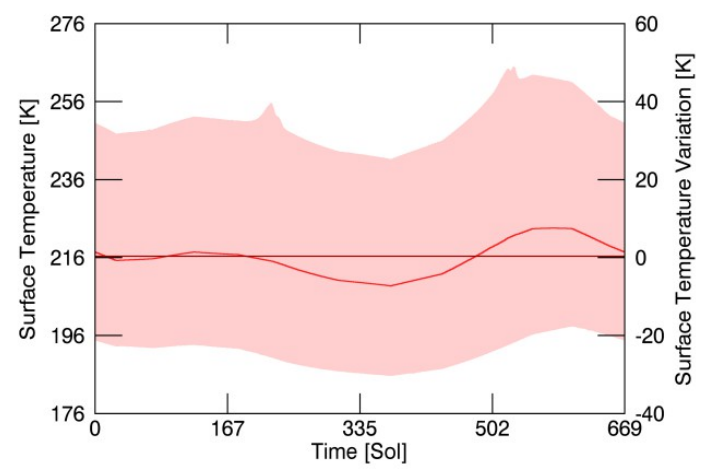

Figure 2. Mars-GRAM simulated surface temperatures for site in the Elysium region within $134^{\circ} \mathrm{E} 3^{\circ} \mathrm{N}$ and $139.5^{\circ} \mathrm{E} 5^{\circ} \mathrm{N}$. The simulation begins on November 1, 2016 (Ls 252.7) and is time stepped at 1 Martian hour over 1 Martian year. The dark line is the mean temperature over 1 Martian year, the midtone curve the mean temperature over $1 \mathrm{Sol}$ and the light-toned curve the calculated temperature plotted every Martian hour. See Mars Climate Database (2013) for guidance on timing used.

A mean surface temperature can be derived from temperature measurements taken over 1 Martian year - this is used for the demonstration purposes herein (Figure 2, dark red line). For shorter measurement periods or scenarios involving transient climatic phenomena (like dust storms), the mean temperature may be obtained from a long term climate simulation which can take into account any probe measurements and minimise the influence of the transient climatic event on the derived mean temperature. Grott et al. (2007) use climate models to show that surface climatic perturbations of the medium- to long-term (>> 1 Martian year) steady temperature should not perturb the Martian heat flow beyond $15 \%$.

\subsection{Thermal Conductivity and Capacity}

A heat flow probe in situ conductivity measurement can be based on the principles of the line heat source technique (or transient hotwire method - e.g. Seiferlin et al., 1996; Banaszkiewicz et al., 1997). The surface conductivity can be estimated from surface thermal inertia (the product of the square root of conductivity and thermal capacity; e.g. Kieffer et al., 1977; Mellon et al., 2000; Christensen et al., 2001). This is achieved by using the known association of thermal inertia with regolith particle size, both of which correlate strongly with thermal conductivity at given temperatures and pressures (Nowicki and Christensen, 2007; Piqueux and Christensen, 2009a,b; Piqueux and Christensen, 2011). 
While Martian thermal properties show some temperature dependence, these are mostly apparent over the large diurnal temperature variation. The focus here on seasonal average thermal properties should mitigate the influence of perturbing effects due to the temperature variation. Thermal properties of the regolith can be assumed to be constant over the annual temperature ranges considered in this model. In cases where temperature dependence is vital to achieving physically meaningful results, the effects of temperature dependence can be replicated by including artificial heat sources or, where the temperature variation is effectively sampled and sufficiently small, centrally estimated parameter values.

Surface conductivities are derived here using measured thermal inertia from sites at $135.10^{\circ} \mathrm{E} 4.37^{\circ} \mathrm{N}$ and $138.23^{\circ} \mathrm{E} 3.31^{\circ} \mathrm{N}$ within the Elysium region identified in Section 3.1; these are fairly high $(0.05$ and $0.07 \mathrm{~W} / \mathrm{m} / \mathrm{K})$ for a fine regolith layer. The preceding result can be interpreted - based on the $<1 \%$ rock abundance $(>25 \mathrm{~cm}$ diameter) results of Golombek et al. (2014) - as rocks smaller than $25 \mathrm{~cm}$ contributing to the sub-pixel thermal inertia signature; though it may also signal a temperature dependent surface thermal conductivity similar to that on the moon (e.g. Heiken et al., 1991). Given these results, regolith properties are assigned based on a silicate sand composition ( $\leq 1 \mathrm{~mm}$ diameter; e.g. Robie et al., 1970; Ruff and Christensen, 2002; Piqueux and Christensen, 2011; Golombek et al., 2013), to give surface conductivities of 0.015 and $0.05 \mathrm{~W} / \mathrm{m} / \mathrm{K}$, corresponding to surface densities of 1100 and $1700 \mathrm{~kg} / \mathrm{m}^{3}$, and a homogeneous specific heat of $700 \mathrm{~J} / \mathrm{kg} / \mathrm{K}$ to produce respective low and high thermal inertia surfaces.

The depth dependence is modelled such that thermal property $m(z)=m_{\infty}(z+a) /(z+b)(\underline{G r o t t}$ et al., 2007): $m_{\infty}$ is the asymptotic thermal property value; constants ${ }^{a}$ and ${ }^{b}$ are chosen such that $m(z)$ equals the surface value at $z=0$ and $m(z)=c m_{\infty}$ at a given depth $(10 \mathrm{~m}) ;{ }^{c}$ is an arbitrary constant. Here, the depth variation represents an idealised fines layer, 
modelled to a thickness of $10 \mathrm{~m}$. The depth profiles for each site are plotted in Figure 3 with the low skin depth site $\left(k_{\infty} \approx 0.07 \mathrm{~W} / \mathrm{m} / \mathrm{K}, \rho_{\infty} \approx 1737 \mathrm{~kg} / \mathrm{m}^{3}\right)$ being highly heterogeneous towards the surface and the high skin depth site $\left({ }_{\infty} \approx 0.12 \mathrm{~W} / \mathrm{m} / \mathrm{K}, \rho_{\infty} \approx 2353 \mathrm{~kg} / \mathrm{m}^{3}\right)$ being nearly homogeneous, with depth.
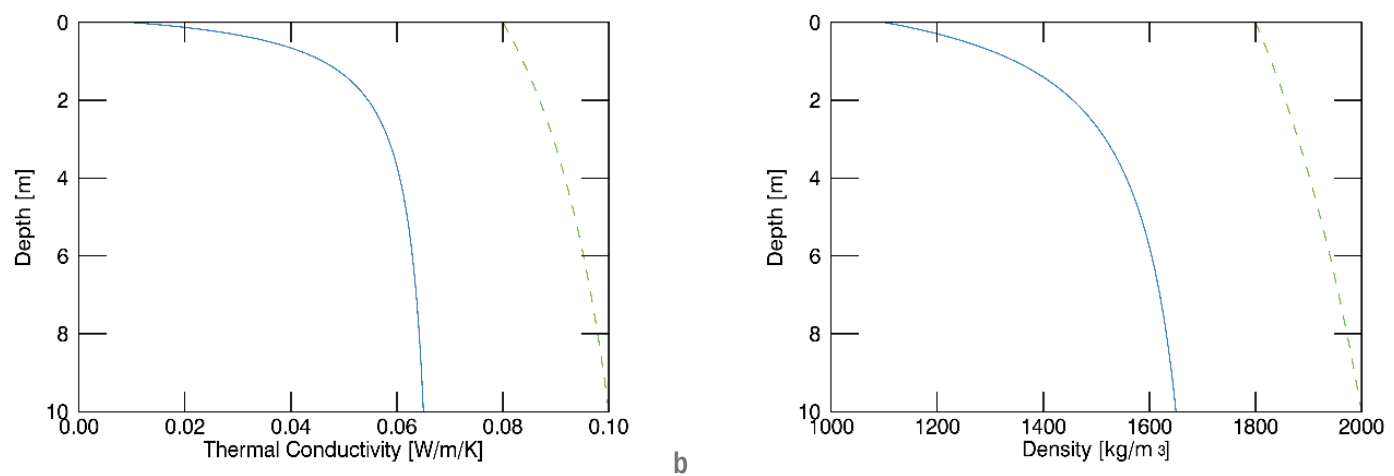

Figure 3. Thermal properties showing: a. conductivity and; b. density for low skin depth (solid blue) and high skin depth (dashed green) heat flow measurement environments representing idealized fines layers.

\section{Synthesised Measurements}

\subsection{Simulation Results}

Simulation results of the low skin depth regolith profile (Figure 3, solid blue) are shown in Figure 4 (left) while results from the high skin depth profile (Figure 3, dashed green) are shown in Figure 4 (right). Each thermal profile is simulated with end member high (25 $\left.\mathrm{mW} / \mathrm{m}^{2}\right)$ and low $\left(5 \mathrm{~mW} / \mathrm{m}^{2}\right)$ pristine background (planetary) heat flows as discussed in Section 3.2 (Figure 4c-f, light grey background plots). In the low skin depth model, a planetary heat flow of $25 \mathrm{~mW} / \mathrm{m}^{2}$ can be directly measured at $2 \mathrm{~m}$ as the gradient of the surface temperature variation is damped to magnitudes within that due to the planetary heat flow; likewise, a planetary heat flow of $5 \mathrm{~mW} / \mathrm{m}^{2}$ can be directly measured at $3.5 \mathrm{~m}$. In the high skin depth model, a planetary heat flow of $25 \mathrm{~mW} / \mathrm{m}^{2}$ can be directly measured at 3.25 $\mathrm{m}$, while that of $5 \mathrm{~mW} / \mathrm{m}^{2}$ can be accessed at $5.25 \mathrm{~m}$. 


\subsection{Temperature Measurements}

Fifteen sensors ( 1 surface +14 subsurface) are used to synthesise measurements from the forward models; the sensor distribution is shown in Figure 4 (grey squares). Temperature measurement errors are simulated with additive Gaussian noise of amplitude 50, 100 and $500 \mathrm{mK}$, the lower extreme related to instrument precision and the higher extreme related to parameter modelling and potential sensor depth inaccuracies.

Independent of the temperature noise level, considered measurement scenarios include sensor penetration depths of approximately 2, 3 and $5 \mathrm{~m}$ and regolith monitoring periods of 0.25 ( vernal equinox), 0.5 ( $\sim$ summer solstice), 0.75 ( autumn equinox) and 1 ( winter solstice) Martian year from Ls 252.7 (based on the original InSight mission profile), along with four instantaneous measurements at the end of the monitoring periods. These scenarios can arise from obstacles such as sedimented layers, large rocks in the regolith and/or instrument failure after a given depth or time period.

The profiles in Figure 4 can be used to visualise all other temperature measurement scenarios: measurements which fall short of one Martian year form sub-profiles of the presented temperature profiles, being cut off as indicated by the coloured (bold) curves (the purple curves also indicate the starting point of all monitoring periods); measurements which fall short of the desired depth are cut off at depth (the grey sensor squares migrate upwards such that the linear array terminates at approximately 2 or $3 \mathrm{~m}$ ); increasing noise levels have random deviations of increasing magnitude from the true profiles.
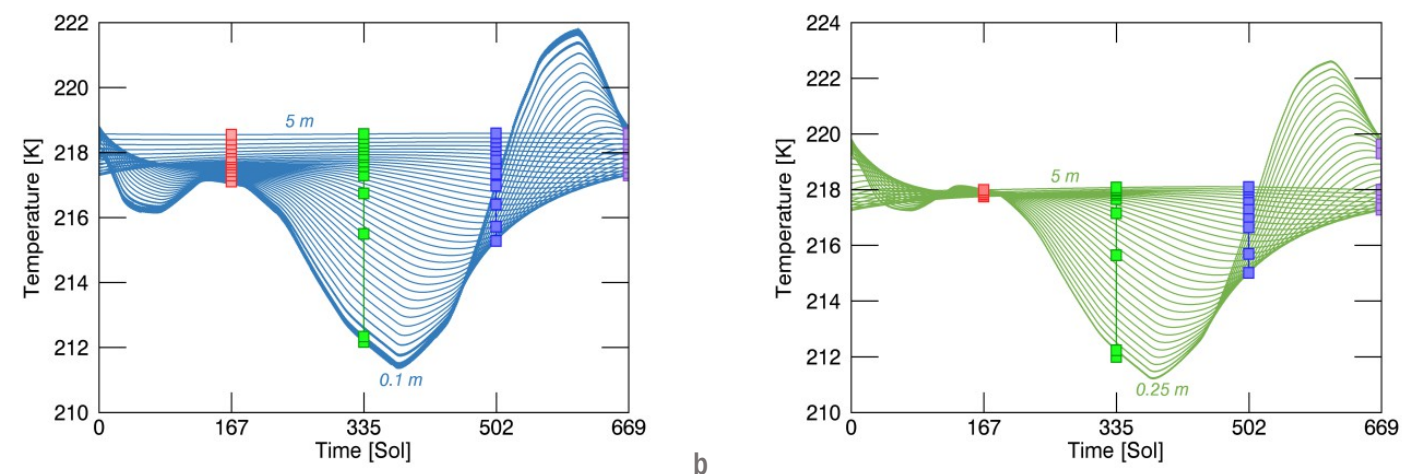

Page 16 of 39 

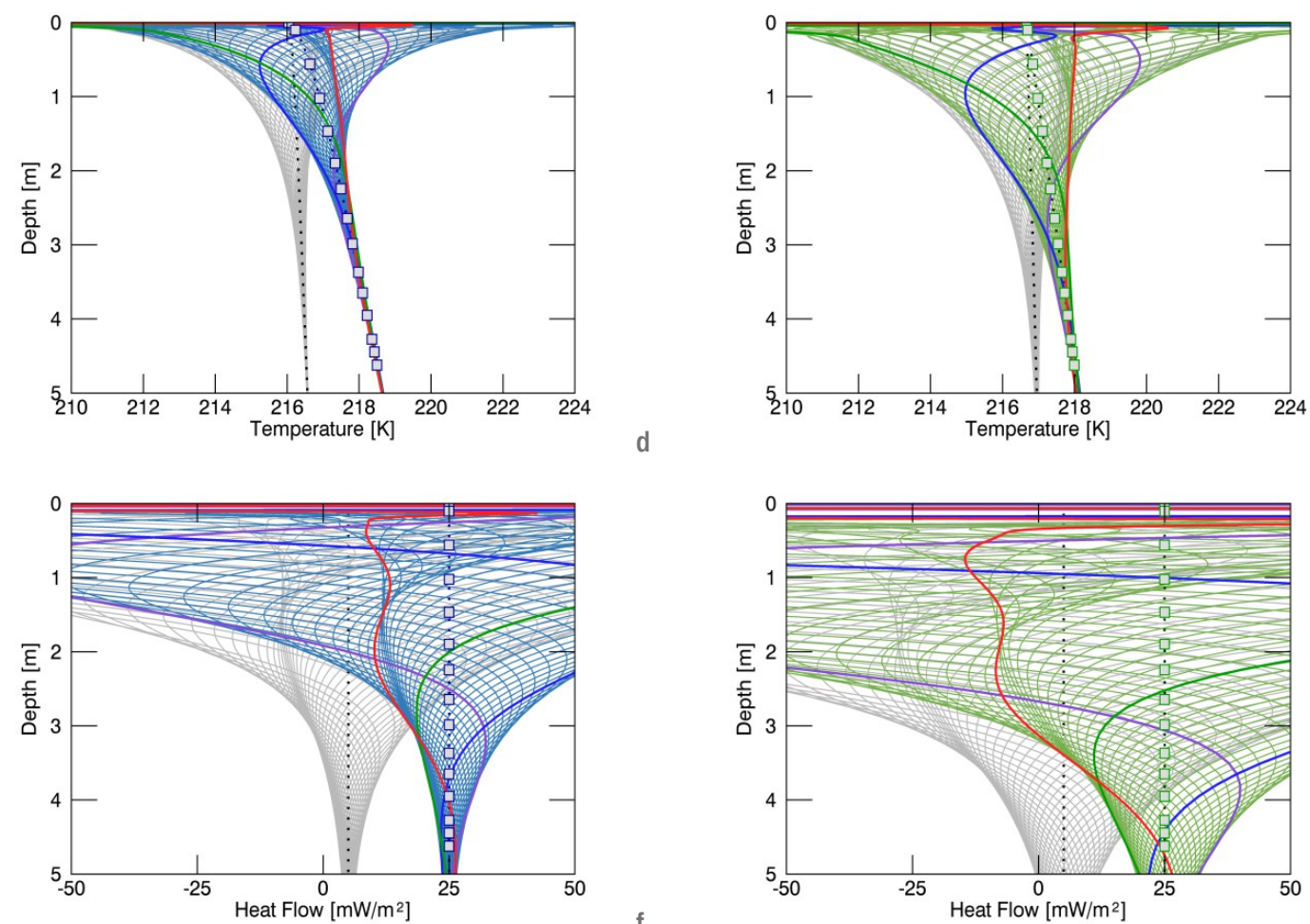

Figure 4. Thermal profiles from Mars Elysium sites with low skin depth (left) and high skin depth (right). Plots a and b show the variation of temperature with time (from Ls 252.7) at different depths (larger amplitudes towards the surface): the coloured squares, which represent sensors, illustrate the location of an instantaneous measurement, or the termination point of a long period measurement. The lower plots show the variation of temperature (c, d) and heat flow (e, f) with depth at different times over a period of $669 \mathrm{Sol}$ in $13.5 \mathrm{Sol}$ steps (overlapping contours indicate periods of relatively constant diurnal mean temperatures): the darker dotted lines running through the contours represent the mean temperature and heat flow; bold coloured lines correspond to instantaneous measurements in a and b; the grey squares show sensor locations, illustrated for a heat flow of $25 \mathrm{~mW} / \mathrm{m}^{2}$; the light background contours show simulations with a heat flow of $5 \mathrm{~mW} / \mathrm{m}^{2}$.

\subsection{Conductivity Measurements}

Conductivity measurements are synthesised with 10-320 \% noise added to the profiles presented in Section 3.4 at depths of approximately 2, 3 and 5 m. These are: Gaussian random noise to produce random errors (10-20\%); negative of the modulus of Gaussian random noise to produce a systematically low estimate (10-50\%); modulus of Gaussian random noise to produce a systematically high estimate (10-320\%); mean of Gaussian random noisy conductivity to assess the viability of using bulk conductivity to estimate the heat flow (10-20\%). The noisy measurement profiles are tested against the true conductivities to assess the effect of the conductivity noise amplitude on the calculated Martian heat flows. 


\section{Heat Flow Estimates}

\subsection{Optimization Scenarios and Heat Flow Solution Space}

The inverse simulations are time stepped in 1 Sol increments over 669 Sol where the diurnal mean surface temperature is applied at each timestep. All other model parameters are used in their pristine forms (Sections 3.3 and 3.4) except for the noisy temperatures, conductivities and heat flows, as outlined in Section 4. The shallowest two sensors are ignored to avoid instabilities introduced by the diurnal temperature variation.

For a select number of measurement scenarios (the primary optimization set) the standard deviations in covariances $C_{m}$ and $C_{d}$ are set such that $10^{-4} \sigma_{d} \leq \sigma_{F_{B}^{s}} \leq 10^{4} \sigma_{d}$, where $0.025 \mathrm{~K}$ $\leq \sigma_{d} \leq_{1} \mathrm{~K}$. These are where the measurement noise amplitude is $0.05 \mathrm{~K}$, monitored over a period of 669 Sol from Ls 252.7 and the conductivities are errorless or with $\pm 20 \%$ systematic noise (high and low estimates). For the full range of measurement scenarios (the secondary optimization set), ${ }_{d}$ is calculated from the temperature errors and related to the heat flow standard deviation by $\sigma_{F_{B}^{s}}={ }_{10} \sigma_{d}$. For all optimizations, the conductivities are assumed accurate and are therefore not included in $m$ and $C_{m}$.
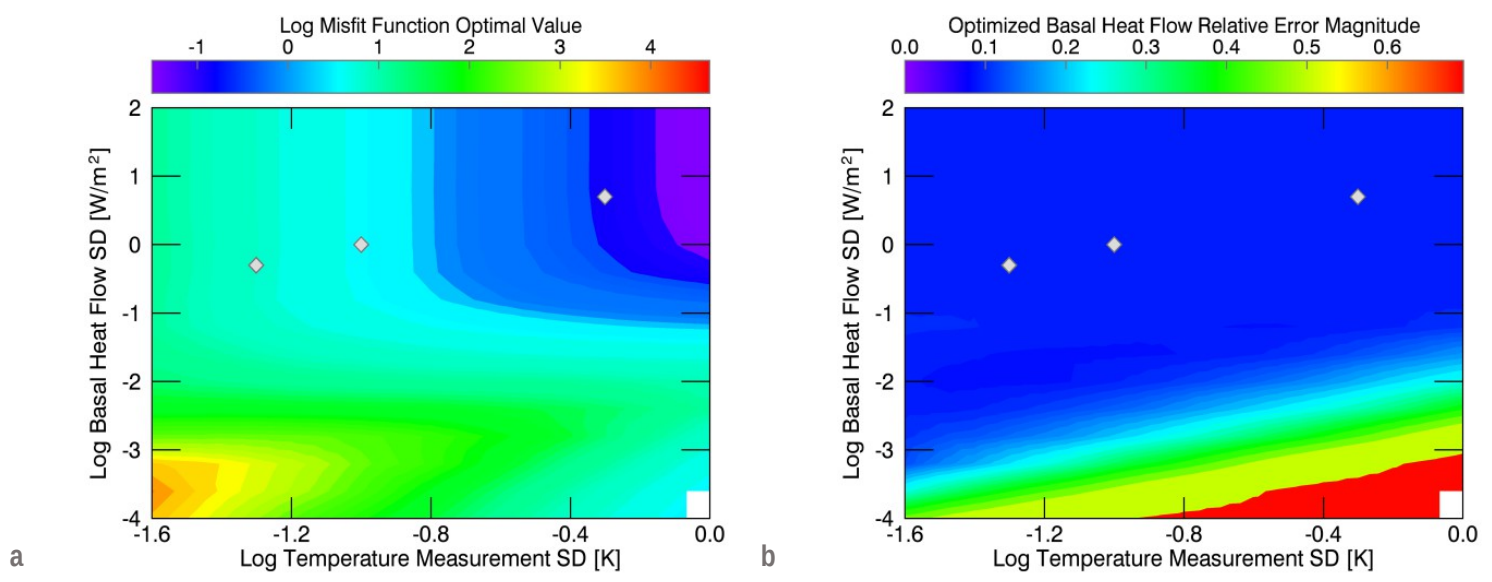

Page 18 of 39 

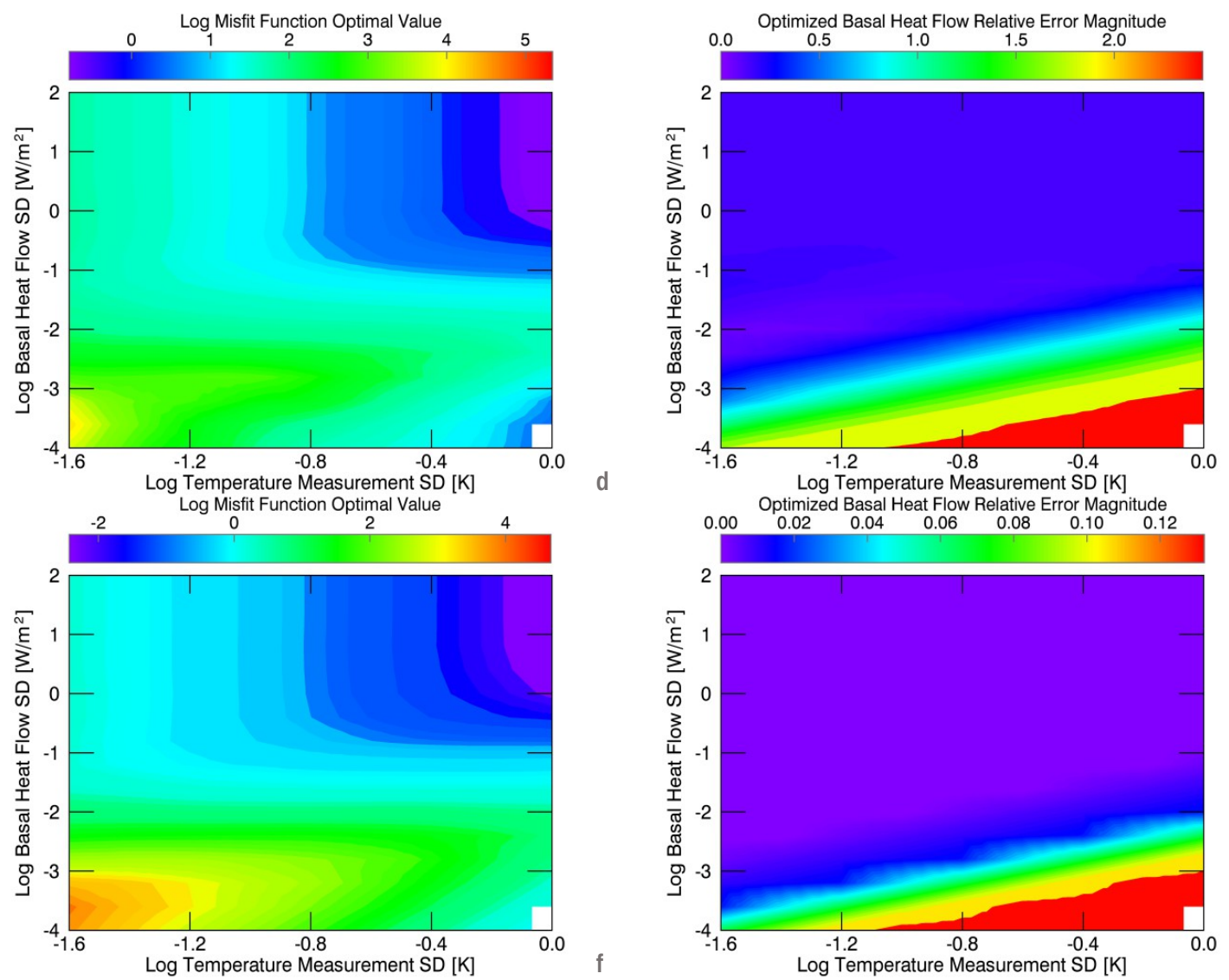

Figure 5. Solutions space defined by standard deviations $\sigma_{F_{B}^{s} \text { and }} \sigma_{d}$ for temperature measurement inversions with misfit function value (left) and heat flow relative error magnitude (right). For each pair: a and b represents a central estimate of the shape of the space over all the tested scenarios - the grey diamonds identify the locations at which the secondary set of scenarios are run; $c$ and $d$ represent the most pathological scenarios (high skin depth with a conductivity systematically high by $20 \%$ and low heat flow of $0.005 \mathrm{~W} / \mathrm{m}^{2}$ ); $\mathrm{d}$. and e. represent the well-behaved scenarios (low skin depth with accurate conductivity and high heat flow of $0.025 \mathrm{~W} / \mathrm{m}^{2}$ ). Notes: 1. there is missing data at the lower right corner of each contour plot; 2 . the contours of plot $f$. are linearly interpolated, while the rest are quintically interpolated.

The inversion results are thus quantifiable with a multi-dimensional results space comprising: the skin depth; depth of measurement; duration and, for instantaneous measurements, time of measurement relative to seasonal period; planetary heat flow; temperature measurement errors; conductivity measurement errors; and in the selection of primary optimization scenarios, covariances. The inverse results are considered acceptable if they fall nominally within the range of the solution space defined by the covariances $C_{m}$ 
and $C_{d}$ (or standard deviations $\sigma_{F_{B}^{s}}$ and ${ }^{\sigma_{d}}$ ), discussed in Section 2.3 (Figure 5; compare Figure 1).

The solution space for the primary set of optimization scenarios, as defined in the $\sigma_{F_{B}^{s}} \sigma_{d}$ plane (Figure 5) shows that the most accurate heat flow solutions can be found in the region of the line which defines $r_{d}^{F_{B}^{s}} \geq 0.1$ (dark blue and violet contours). Above the $r_{d}^{F_{B}^{s}}$ boundary, all optimized heat flows show significant improvements in accuracy on the initial estimates in nearly all measurement scenarios. Those with least accuracy (Figure 5c-d) have high skin depth, systematically high thermal conductivity and low pristine heat flow. Conversely, the most accurate scenarios (Figure $5 \mathrm{~d}$-e) have low skin depth, accurate thermal conductivity and high heat flow. Note that the secondary optimization scenarios (grey diamonds in Figure $5 a-b)$ are all run within the heat flow solution space; the rest of the discussion takes account of results from both the primary and secondary set of optimization scenarios.

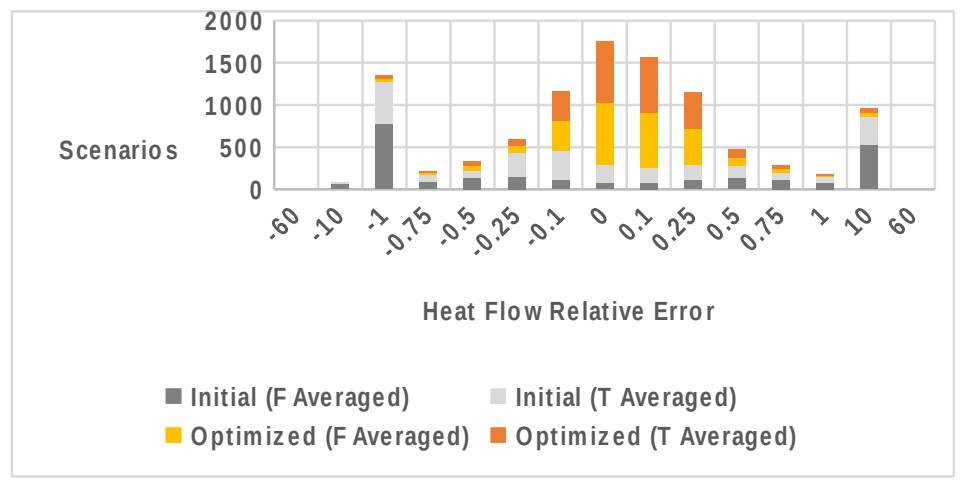

Figure 6. Distribution of heat flow relative error for scenarios with initial heat flows calculated using method 1 (lower middle bars) and method 2 (base) and corresponding optimized distributions. Each bar represents the number of scenarios with errors greater than the previous error value, up to and including the value under the bar (e.g. the bar at -0.1 represents values in the interval $(-0.25,-0.1]$. These results include conductivities with Gaussian and systematic errors up to $20 \%$.

Initial heat flow estimates, calculated directly from the mean temperatures (method 1 ) are, on average, $61 \%$ more accurate than those calculated with the mean heat flows from each timestep (method 2). The latter heat flows are, on average, incorrect by a factor of $3.6-$ 
within the range theorised in Section 2.4. Heat flow values calculated by the different methods are the same for instantaneous measurements, as predicted. The optimization results are approximately the same for the background heat flow, regardless of which initial estimate is used (Figure 6), therefore, for brevity, only results using method 1 are further presented. The distribution of results in Figure 6 shows the general improved accuracy of the initial (direct) heat flow estimates calculated using method 1 (T Averaged) over those calculated using method 2 (F Averaged). Optimization substantially modifies the initial heat flow distribution such that it peaks where the relative error is close to zero.

\subsection{Accurate and Noisy Conductivity}

\subsubsection{General Results and Depth of Measurement}

Where the conductivity-depth profile is accurate (but potentially contains noise up to $20 \%$ ), the initial heat flow accuracy is significantly improved by optimization in most measurement scenarios. Exceptions occur at the shallowest sensor depths for high skin depth, instantaneous measurement or short monitoring period (0.25 Martian years) scenarios; in these scenarios the influence of the temperature variation is large such that the initial heat flow estimate is already of optimal accuracy for the given parameter set. Generally, with basal sensor depths at $2.13,3.28$ and $4.6 \mathrm{~m}$, heat flows are respectively optimized to accuracies of 29,7 , and $4 \%$ (accordingly 10,34 and 38 times more accurate than the direct estimates). The conductivity noise up to $20 \%$ has a relatively negligible impact on the heat flow accuracy.

Page 21 of 39 


\subsubsection{Instantaneous Measurements}

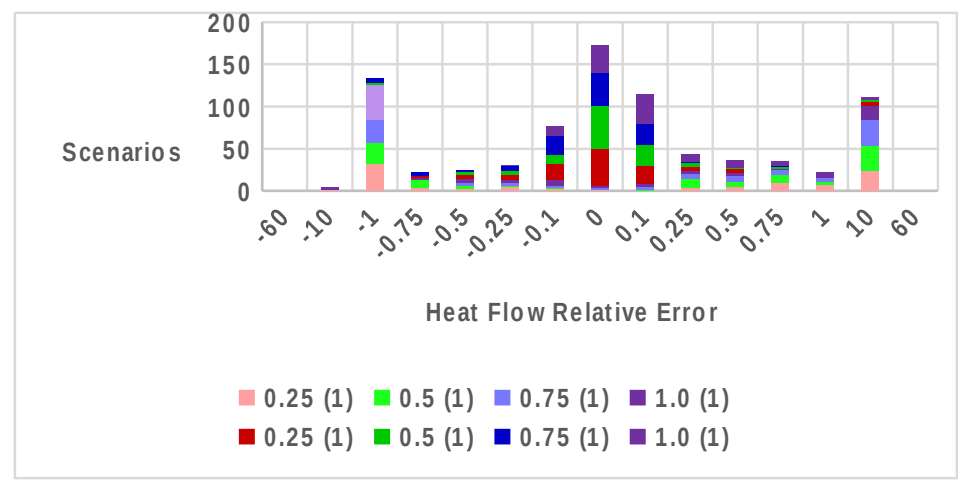

Figure 7. Distribution of heat flow relative error for instantaneous measurement scenarios. The four lower (light coloured) bars represent initial errors and the four upper (dark coloured) bars represent errors after optimization for the relevant measurement time after Ls 252.7 in Martian years. Each bar represents the number of scenarios with errors greater than the previous error value, up to and including the value under the bar (e.g. the bar at -0.1 represents values in the interval $(-0.25,-0.1]$. These results include conductivities with Gaussian errors up to $20 \%$.

The accuracy of heat flow optimized from an instantaneous measurement is particularly sensitive to the shape of the measurement in terms of the temperature gradient due to the temperature variation, the skin depth relative to the sensor depth, and the rate of change of the surface temperature at and prior to the time of measurement. For example, measurements taken at 0.5 Martian years ( summer solstice), on average, yield the most accurate optimized heat flows (17\%; 25 times more accurate than the corresponding direct estimate) while measurements taken at 0.75 Martian years ( autumn equinox) are, on average, optimized to an accuracy of $26 \%$ (roughly 17 times the accuracy of the direct estimate). With the former measurement, temperature increases monotonically with depth while the latter has more oscillations and a steeper surface temperature gradient (Figure 4ad). Figure 7 shows the distribution of results, illustrating a bias towards higher optimized heat flows from measurements at 1 Martian year ( winter solstice), and the reverse for measurements at 0.75 Martian years; this can be traced back to the respective temperature gradients underestimating and overestimating the background heat flow (see Figure 4c-d). 


\subsubsection{Long Period Measurements}

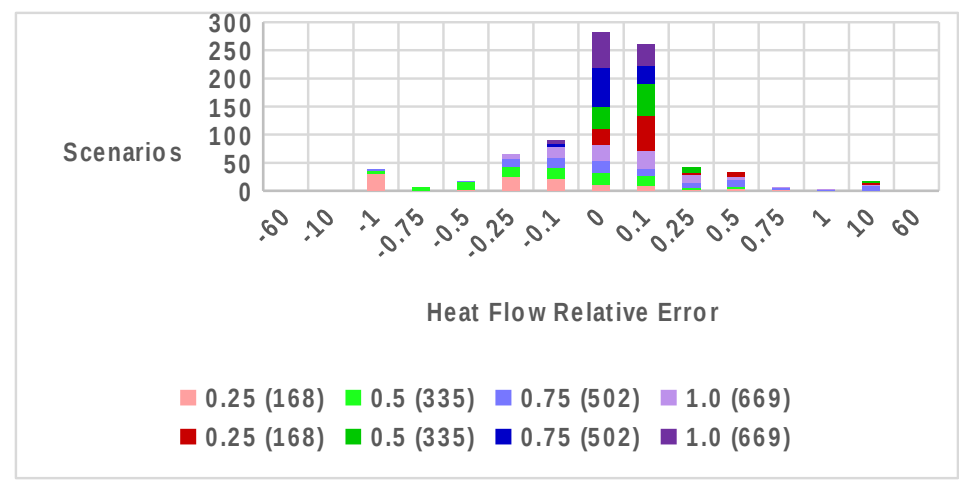

Figure 8. Distribution of heat flow relative error for long period measurement scenarios. The four lower bars represent initial errors and the four higher bars represent errors after optimization for the relevant measurement period after Ls 252.7 in Martian years. Each bar represents the number of scenarios with errors greater than the previous value, up to and including the error value under the bar (e.g. the bar at -0.1 represents values in the interval $(-0.25,-0.1]$. These results include conductivities with Gaussian errors up to $20 \%$.

Where the regolith is monitored over long periods (0.25-1 Martian year), the optimized heat flow accuracy increases with the length of the monitoring period (11-3\%, on average, approximately 5 times the corresponding direct heat flow accuracy). There is appreciable divergence from the former trends for the direct heat flow estimates. For example, for a monitoring period of 0.75 Martian years inaccuracies are higher than the corresponding values for 0.5 Martian years. For the optimized heat flows, at $4.6 \mathrm{~m}$ there is no dependence of the heat flow accuracy on monitoring period, the cause of which can be traced back to the damping of the temperature variation at depth, such that instabilities are introduced only by the temperature measurement noise. Figure 8 shows the distribution of results, which are markedly less pathological than those for the instantaneous measurements, demonstrating the importance of a monitoring period of appropriate length. 


\subsubsection{Temperature Measurement Errors}

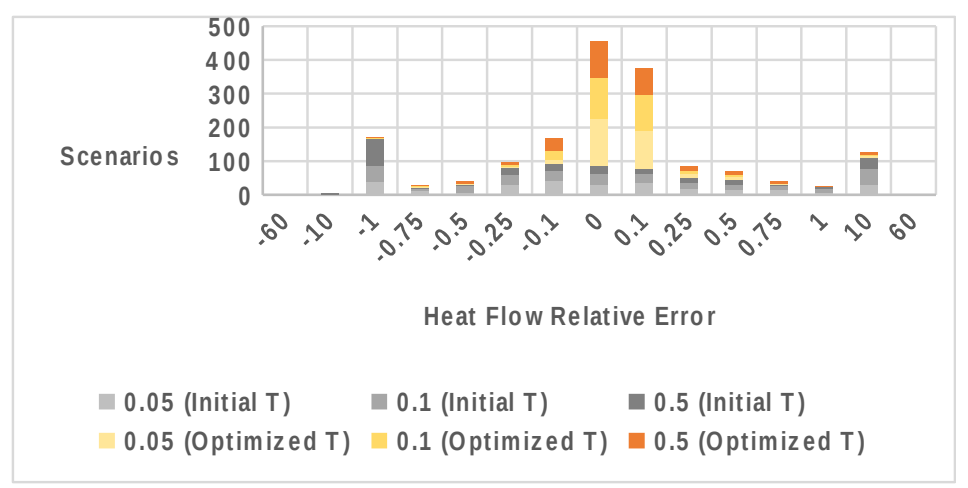

Figure 9. Distribution of heat flow relative error for temperature measurements with varying precision errors. Each bar represents the number of scenarios with errors greater than the previous value, up to and including the error value under the bar (e.g. the bar at -0.1 represents values in the interval $(-0.25,-0.1]$. These results include conductivities with Gaussian errors up to $20 \%$.

The temperature measurement errors of $50-500 \mathrm{mK}$, on average, produce optimized heat flows accurate to $10 \%, 10$ times more accurate than the corresponding directly measured heat flows. The rate of increase of the heat flow errors with temperature errors is smaller for the optimized heat flows such that for the $50 \mathrm{mK}$ errors, the accuracy of the optimized heat flow estimate improves from $87 \%$ to $9 \%$ (factor of 9.67) while for the $100 \mathrm{mK}$ errors, the corresponding figures are $113 \%$ to $11 \%$ (factor of 10.27). If $500 \mathrm{mK}$ errors are included that factor increases to 24.6 (468 \% to $19 \%$ ). Figure 9 illustrates the distribution, which shows significant correlation between the different precision errors, indicating a uniform response of the algorithm to the different noise levels in the measurements. 


\subsubsection{Pristine Planetary Heat Flow}

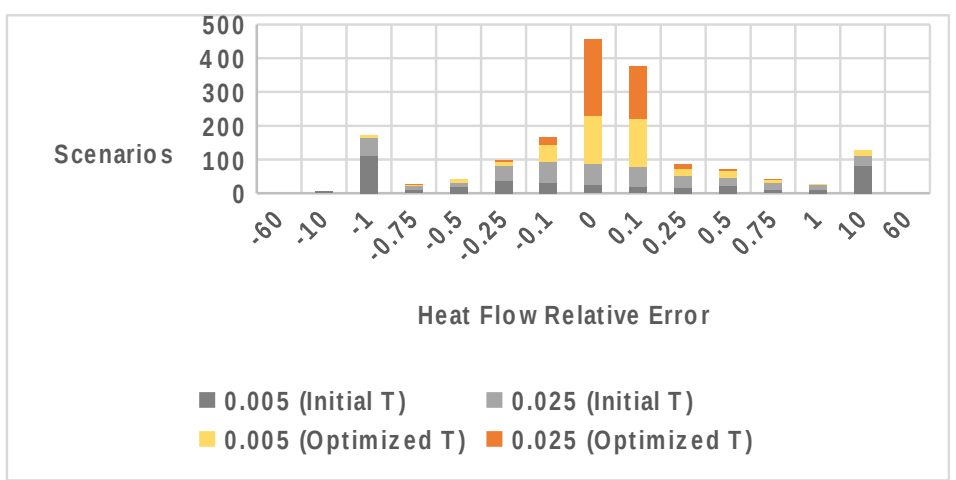

Figure 10. Distribution of heat flow relative error for different pristine heat flow environments. Each bar represents the number of scenarios with errors greater than the previous value, up to and including the error value under the bar (e.g. the bar at -0.1 represents values in the interval $(-0.25,-0.1]$. These results include conductivities with Gaussian and systematic errors up to 20 $\%$.

Given higher pristine planetary heat flow, the heat flow estimates are recovered with greater accuracy, as suggested in Section 4.1. For instantaneous measurements, optimized heat flow estimates are, on average, accurate to $7 \%$ at $25 \mathrm{~mW} / \mathrm{m}^{2}$ and $35 \%$ at $5 \mathrm{~mW} / \mathrm{m}^{2}$; roughly 20 times more accurate than the direct estimates. For long-period measurements, heat flows are, on average, optimized to approximately $3 \%$ accuracy at $25 \mathrm{~mW} / \mathrm{m}^{2}$ and $9 \%$ at 5 $\mathrm{mW} / \mathrm{m}^{2}$, respectively 3 and 7 times more accurate than the direct estimates. The distribution in Figure 10 illustrates the relatively pathological nature of the low heat flow environment.

\subsubsection{Skin Depth}

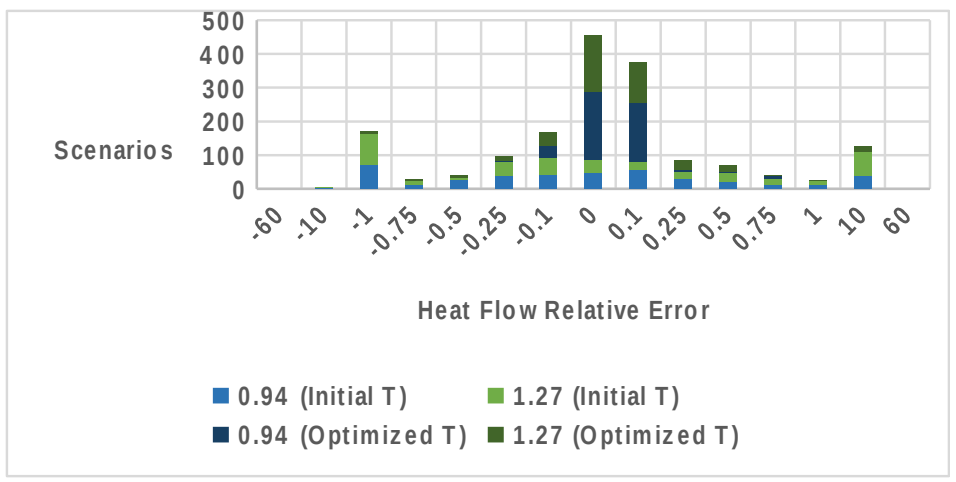


Figure 11. Distribution of heat flow relative error for different skin depth environments. Each bar represents the number of scenarios with errors greater than the previous value, up to and including the error value under the bar (e.g. the bar at -0.1 represents values in the interval $(-0.25,-0.1]$. These results include conductivities with Gaussian errors up to $20 \%$.

Naturally, the lower skin depth scenarios generally provide more accurate initial and optimized heat flow estimates, where the deeper measurements provide the more accurate heat flow estimates. Directly measured, the heat flow at low skin depth $(0.94 \mathrm{~m})$ is, on average, accurate to within $192 \%$ while optimization increases the accuracy to $5 \%$. At high skin depth $(1.27 \mathrm{~m})$, the former figures are revised upwards to $253 \%$ and $21 \%$. Figure 11 illustrates the distribution of results.

Note that the results relative to each parameter discussed above are filtered only with respect to the specific parameter, and conductivity. For example, if heat flow magnitude is considered in the skin depth results: at low skin depth the heat flows are, on average, optimized to an accuracy of $2 \%$ at $25 \mathrm{~mW} / \mathrm{m}^{2}$ pristine heat flow, while at high skin depth the heat flows are, on average, accurate to $35 \%$ at $5 \mathrm{~mW} / \mathrm{m}^{2}$. The preceding figures represent improvements on the direct estimates by factors of 33 in the former case and 12 in the latter. The evolution of heat flow estimates with respect to the preceding parameters are shown in Appendix B.

\subsection{Systematic Conductivity Errors}

Systematically inaccurate conductivities introduce instabilities, with results depending on the profile of the conductivity. For example, for the low skin depth scenario, where the conductivity changes rapidly at shallow depth, using bulk conductivity leads to the most inaccurate optimized results (30\%), while for the high skin depth scenario with near homogeneous conductivity, using bulk conductivity is more permissible (10\% error after optimization). These results are illustrated in Figure 12, where the bulk conductivities in the low skin depth cases (left plots) produce the largest deviations from the true temperature gradient. Generally, overestimated conductivity (with a resulting low temperature gradient) leads to overestimated optimized heat flow, and vice versa (also see Figure 13). 

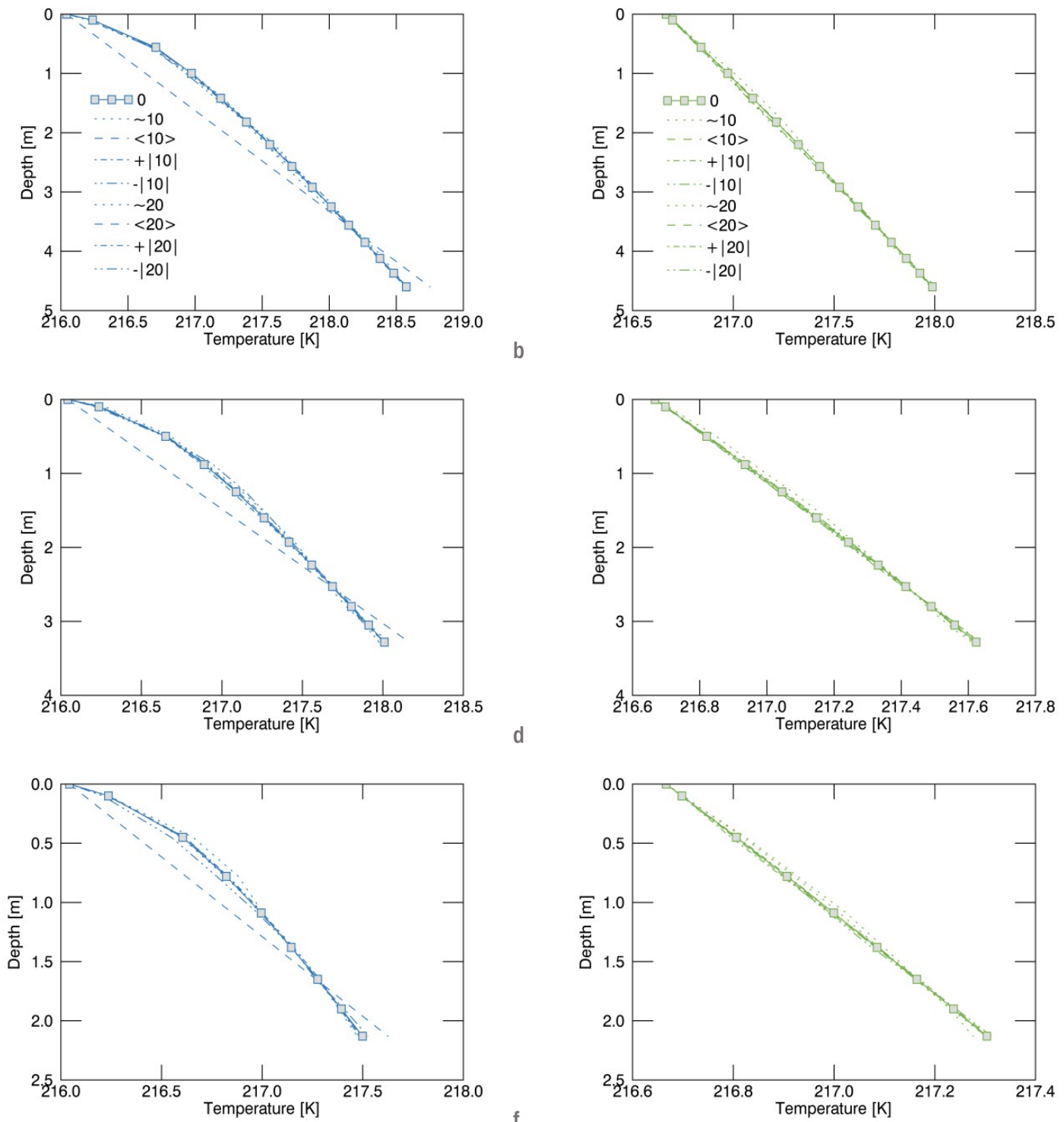

Figure 12. Background temperature profile results for temperature measurement inversions with low skin depth $(0.94 \mathrm{~m})$ scenarios (left) and high skin depth (1.27 m) scenarios (right). The upper middle and lower pairs represent measurement depths of $4.6,3.28$, and $2.13 \mathrm{~m}$, respectively. The different curves (see legends) show results for conductivities with no errors (0), and respective mean $(<>)$, Gaussian random $(\sim)$, systematic high $(+||)$ and systematic low $(-||)$ errors for $10 \%$ and $20 \%$ noise amplitudes. The grey squares are sensor locations.

More specifically, where conductivity estimates are high by $10-20 \%$, heat flows are optimized to accuracies in the range $19-39 \%$, on average, while similarly underestimated conductivities produce heat flow estimates which are respectively $20-24 \%$ inaccurate. The preceding figures are in contrast to conductivities with accurate, but noisy profiles where conductivity noise of $10-20 \%$ produces optimization errors in the range $9-24 \%$. The former cases are typically 7-25 times more accurate than corresponding direct estimates. 


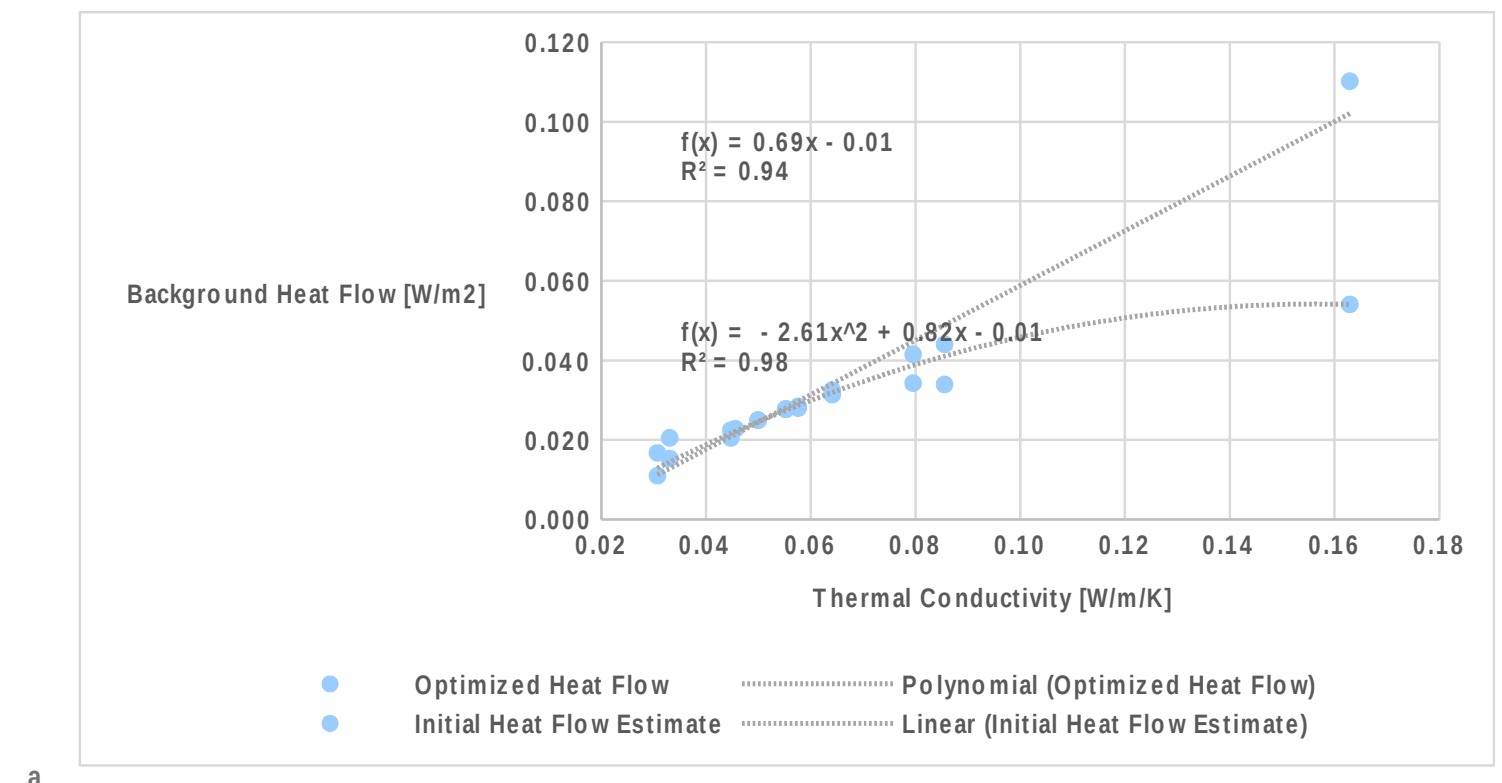

a

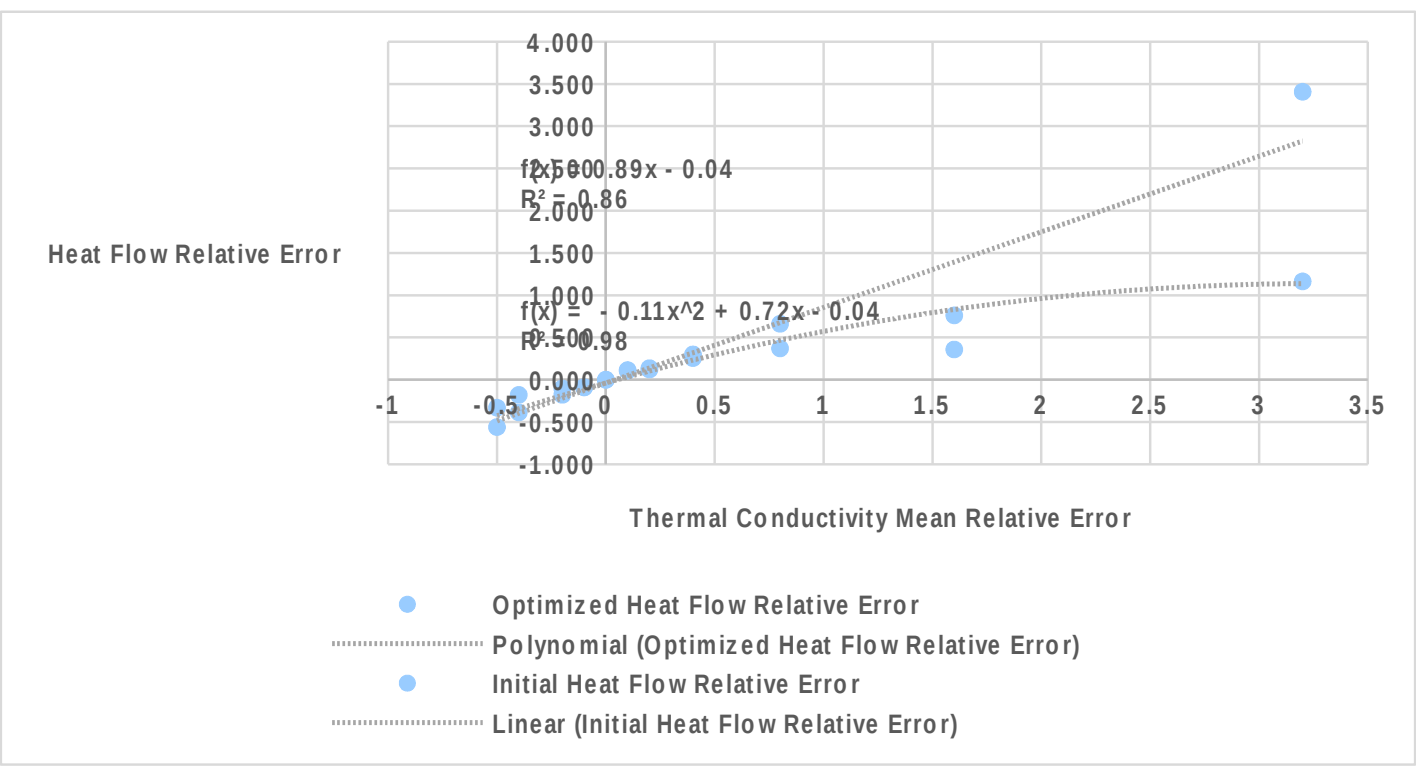

b

Figure 13. Heat flow estimates (a) and relative errors (b) relative to thermal conductivity estimates and relative errors. These are for long period subsurface temperature measurement scenarios taken at 1 Martian year from Ls 252.7, with measurement depth $4.6 \mathrm{~m}$, errors up to $50 \mathrm{mK}$, skin depth of $0.94 \mathrm{~m}$ and a pristine heat flow of $25 \mathrm{~mW} / \mathrm{m}^{2}$. Note that the bulk conductivities and those with Gaussian noise up to $20 \%$ are not included in the plots.

Data for conductivities with systematic errors larger than $20 \%$ are produced only for measurements monitored over (or taken at) 1 Martian year from Ls 252.7 at $4.6 \mathrm{~m}$ depth, with $50 \mathrm{mK}$ temperature errors (Figure 13). Where conductivities are high by $40-320 \%$ the heat flow inaccuracies are in the range 34-131\% (optimized) and 56-219\% (direct). Conductivities low by 40-50\% produce heat flows with errors in the range 40-55\% (optimized) and $37-46 \%$ (direct). Note that the direct estimates approach a 1:1 relationship 
with the conductivity errors as the influence of temperature measurement errors and the temperature variation are minimised.

Direct heat flow estimates have similar tolerance to systematic conductivity errors up to \pm 40 $\%$ and optimized heat flow estimates, likewise, up to $\pm 20 \%$ (Figure 13). The preceding result also holds with the use of bulk conductivities. Figure 13 shows that heat flows are optimized to a limiting value (while direct estimates increase linearly) with increasing systematic conductivity errors; this behaviour holds for any given scenario, with variation in the curve parameters (equation) based on that of the model parameters across individual scenarios (pristine heat flow, monitoring period, skin depth, temperature measurement errors).

\section{Discussion}

\subsection{Scenarios}

The results show that inverting a depth-resolved subsurface temperature measurement can, in the majority of tested scenarios, lead to planetary heat flow estimates more accurate than those directly determined from the temperature gradient and estimated thermal conductivities. Calculating heat flow by taking the mean temperature across several measurements over a given period generally gives more accurate estimates than calculating heat flows from individual measurements, then averaging them; this is because the temperature measurement errors tend to be averaged out in the former method, whereas they are amplified in the latter. The opposite happens with conductivity errors, therefore a comparative assessment is required where the conductivity errors are significant - further, using both calculation methods can deliver insight into the relative importance of the contributing error parameters.

Optimization increases the accuracy of direct estimates by factors between 1-1000, depending on the characteristics of the measurement scenario. Direct estimates have accuracies between 0-444 \%, likewise, optimization between 0-478\% - the critical 
difference being, as Figure 6 shows, the Gaussian distribution about $0 \%$ for the optimized heat flows. Optimization tends to provide the most useful results with instantaneous or short period measurements taken at the shallowest depths, where direct estimates typically achieve poor results. The overall results demonstrate a $52 \%$ chance of achieving a direct heat flow estimate more accurate than $40 \%$ (using the temperature averaging method) with the same being $82 \%$ after optimization.

Directly calculated, the heat flow increases linearly with higher assumed bulk conductivity, while optimized, the heat flow estimates tend to a limiting value with higher bulk systematic errors in the assumed conductivity, as illustrated in Figure 13; optimization with unknown conductivity can therefore be used to place bounds on the true conductivity profile. The background heat flow estimate is also less sensitive to temperature precision errors with optimization, which can be gathered from the columns of Table 1 in Appendix B. The reduced sensitivity to errors in optimization is due to the constraining effect of the other model parameters (boundary and internal conditions), therefore, the other parameters must be robustly known for the behaviour to be used effectively.

Use of bulk conductivity is permissible where the conductivity profile is relatively homogeneous over a given depth; if there is substantial variation, use of the bulk conductivity is likely to produce relatively inaccurate heat flow estimates over said depth, as evidenced in Figure 12. Precision errors in conductivity have a negligible impact given an accurate depth-profile. The conductivity depth-profile can be discerned from the mean temperature gradient with depth, given the regolith is successfully monitored over one or more Martian years.

Longer monitoring periods and deeper measurements lead to more accurate heat flow estimates in general. Optimized heat flows are less sensitive to monitoring period in the tested scenarios of 0.25-1 Martian year after Ls 252.7, though sensitivity increases with exposure to the temperature variation through higher skin depth or shallower measurement depth. For instantaneous measurements, the heat flow estimates depend on the degree of 
masking of the mean subsurface temperature by the temperature variation; results indicate that the most accurate optimized heat flows can be expected from measurements taken around the vernal equinox (0.25 Martian years from Ls 252.7) or towards the summer solstice (0.5 Martian years from Ls 252.7 ) where the temperature variation is low.

\subsection{Methodology}

The initial heat flow estimates, obtained directly from the temperature gradient and conductivity, are applied here in a strictly generalised sense - i.e. the heat flow is calculated in exactly the same manner for all measurements, as noted in Section 5.1, to achieve a consistent bulk analysis of the results. For any particular measurement scenario (instantaneous measurements, in particular), the use of the two lowest sensors to calculate the heat flow may not be the best approach; the use of more or different sensors may be more appropriate.

The inverse parametrization used herein allows the solution space of a given scenario to be systematically characterized with a range of standard deviations and covariance operators; previous approaches with the method appear to involve a mixture of trial and error and educated guessing to identify suitable standard deviation values. The space of standard deviations permits the identification of a distinct subspace from which viable solutions can be taken. The relative error of the basal heat flow is used here to effectively illustrate the errors; however, in a real scenario this is not available. Therefore, the values of the heat flow estimates and/or the form of the misfit function space require examination to identify the region of the solution space, as discussed in Section 2.3 (also see Figure $5 \mathrm{a}, \mathrm{c}$ and e). Effectively, for heat flow, the solution space can be found for any given value of standard deviation for the temperature measurement errors by varying the value of the heat flow standard deviation.

The most ill-determined problems may not provide any physically meaningful solutions within a given solution space. This usually points to significant systematic errors in the model 
parameters or, a flaw within the model itself; for example, where the temperature dependence of the model parameters is significant. The effects of temperature dependence can be simulated by the introduction of time- and depth-dependent regolith heat sources in the primal heat flow equation, though the complex effects may be difficult to reproduce. The method presented here is useful where the perturbing effects of temperature dependence can be minimised - it would benefit from an extension of the theory to explicitly account for temperature dependence.

It is important to note that the work presented here assumes a horizontally homogeneous medium, such that the background heat flow determined corresponds to the planetary heat flow from the interior. Extrapolating the estimated background heat flow to a regional or global mean value requires further work similar to that presented in Grott and Breuer (2010) which takes account of lateral inhomogeneity across the Martian surface. The global estimates presented therein can, for example, be normalized to the local measurement to provide an updated global mean estimate of Martian heat flow.

\section{Appendices}

\section{A. Gradient of the Misfit Function}

The gradient $Y$ is found by analytical development of the duals (e.g. Shen and Beck, 1991; Tarantola, 2005) of Equations -. The dual equations take the same form as Equations - (the

primals), except with a reversal of the time component of Equation to $-\rho c(z) \frac{\partial T^{D U}(z, t)}{\partial t}$ and all boundary parameters homogeneous ( ${ }^{D}$ superscript distinguishes the dual parameters). By setting the source terms and dependent variables of the primal-dual pairs as mutual 
duals, such that the scalar product $\left\langle T^{D U}(z, t), S^{U}(z, t)\right\rangle$ is set equivalent to $\left\langle S^{D U}(z, t), T^{U}(z, t)\right\rangle$ and likewise $\left\langle T^{D S}(z), S^{S}(z)\right\rangle-\left\langle S^{D S}(z), T^{S}(z)\right\rangle=0, T^{D U}(z, t)$, the dual temperature variation, becomes equivalent to $S^{U}(z, t)$ in Equation (and $T^{U}(z, t)$ to $S^{D U}(z, t)$ ) with the same following in Equation .

The homogeneous boundary conditions of the dual equations allow straight forward analytical development of the dual of $\frac{\partial d}{\partial m} \equiv \frac{\partial g(m)}{\partial m}$ in $\gamma$; calculation of $\frac{\partial d}{\partial m}$ is usually nontrivial (e.g. Wang, 1992). With the equivalence of the primal temperature and dual source, a perturbation $\delta d=g(m)-d_{0}$ in misfit function $\sum$ (Equation ) represents a perturbation $\delta T(z, t)$ in the temperature and $\delta S^{D}(\mathrm{z}, t)=\delta S^{D U}(\mathrm{z}, t)+\delta S^{D S}(\mathrm{z})$ in the dual heat sources, which gives $\delta T^{D}(\mathrm{z}, t)$ upon solution of the duals of Equations -. The dual of $\gamma$ can therefore be calculated with the dual of $\frac{\partial d}{\partial m}$, from which $Y$ can be obtained by application of a weighting operator; in the quasi-Newton procedure, the latter is the inverse of the dual of the Hessian of $\Sigma, \frac{\partial \gamma}{\partial m}$.

For the planetary heat flow, $\frac{\partial d}{\partial F_{B}^{S}}=-T^{D S}$. 


\section{B. Evolution of Heat Flow Estimates Relative to Monitoring Period, Temperature Precision, Measurement Depth and Skin Depth}

Table 1 shows a selection of the model data (with initial heat flow directly calculated from the mean temperature and excluding $500 \mathrm{mK}$ temperature modelling errors). Table 1 and the presentation of parameter-specific results in Section 5.2 illustrate which parameters contribute to different peaks and trends in the general distribution of results in Figure 6 . It can be gathered, for example, that optimization results with heat flow relative error between 0.75-10 typically reflect high skin depth scenarios with instantaneous measurements taken at 0.75 Martian years up to $2.13 \mathrm{~m}$ depth. 
Table 1. Relative errors in heat flow for the low skin depth measurement (blue, upper) and high skin depth measurement (green, lower) across conductivity scenarios with 10-20 \% Gaussian and systematic error, temperture measurements with 50-100 mK errors and all pristine heat flow scenarios. For each pair of errors shown, the left value is that for the heat flow calculated from the temperature and thermal conductivity while the right value (shaded cells) is for the optimized heat flow estimate. Time is measured from Ls 252.7.

\begin{tabular}{|c|c|c|c|c|c|c|c|c|c|c|c|c|c|c|c|c|c|}
\hline \multirow{4}{*}{ 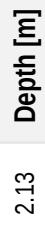 } & \multicolumn{8}{|c|}{ Instantaneous Measurement Time [Martian Years] } & \multicolumn{8}{|c|}{ Measurement Monitoring Period [Martian Years] } & \multirow{3}{*}{$\begin{array}{l}\text { m } \\
\text { 음 } \\
\text { 좆 }\end{array}$} \\
\hline & \multicolumn{2}{|c|}{0.25} & \multicolumn{2}{|c|}{0.5} & \multicolumn{2}{|c|}{0.75} & \multicolumn{2}{|c|}{1} & \multicolumn{2}{|c|}{0.25} & \multicolumn{2}{|c|}{0.5} & \multicolumn{2}{|c|}{0.75} & \multicolumn{2}{|c|}{1} & \\
\hline & 0.83 & 0.16 & 2.34 & 0.20 & 2.50 & 0.56 & 3.25 & 0.20 & 0.75 & 0.18 & 0.37 & 0.14 & 0.21 & 0.10 & 0.18 & 0.13 & \\
\hline & 0.56 & 0.16 & 1.14 & 0.19 & 0.80 & 0.56 & 1.35 & 0.19 & 0.69 & 0.18 & 0.38 & 0.14 & 0.18 & 0.10 & 0.16 & 0.13 & 0.05 \\
\hline \multirow{2}{*}{ 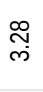 } & 1.64 & 0.12 & 1.02 & 0.10 & 2.61 & 0.21 & 1.50 & 0.11 & 0.34 & 0.06 & 0.37 & 0.06 & 0.15 & 0.08 & 0.14 & 0.10 & 0.1 \\
\hline & 0.67 & 0.07 & 1.24 & 0.08 & 1.56 & 0.21 & 0.40 & 0.08 & 0.28 & 0.06 & 0.26 & 0.06 & 0.18 & 0.08 & 0.15 & 0.10 & 0.05 \\
\hline \multirow{3}{*}{$\stackrel{0}{+}$} & 1.74 & 0.07 & 0.53 & 0.09 & 2.86 & 0.12 & 0.48 & 0.09 & 0.14 & 0.08 & 0.12 & 0.08 & 0.11 & 0.09 & 0.11 & 0.09 & 0.1 \\
\hline & 0.90 & 0.07 & 0.33 & 0.08 & 0.76 & 0.12 & 0.57 & 0.10 & 0.11 & 0.08 & 0.14 & 0.08 & 0.11 & 0.09 & 0.11 & 0.09 & 0.05 \\
\hline & \multicolumn{8}{|c|}{$\Delta$ LOW SKIN DEPTH $[0.94 \mathrm{~m}] \boldsymbol{\Delta}$} & \multicolumn{8}{|c|}{$\boldsymbol{\nabla}$ HIGH SKIN DEPTH $[1.27 \mathrm{~m}] \boldsymbol{\nabla}$} & \\
\hline \multirow{2}{*}{$\stackrel{m}{\sim}$} & 0.55 & 0.43 & 1.61 & 0.50 & 0.70 & 1.00 & 4.07 & 0.81 & 1.04 & 0.56 & 0.13 & 0.32 & 1.00 & 0.11 & 0.17 & 0.07 & 0.1 \\
\hline & 2.14 & 0.38 & 1.71 & 0.48 & 1.00 & 0.98 & 5.77 & 0.84 & 0.92 & 0.56 & 0.13 & 0.32 & 0.97 & 0.11 & 0.12 & 0.07 & 0.05 \\
\hline \multirow{2}{*}{ 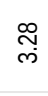 } & 2.21 & 0.15 & 3.55 & 0.06 & 0.68 & 0.38 & 2.11 & 0.08 & 0.91 & 0.11 & 0.52 & 0.10 & 0.28 & 0.03 & 0.10 & 0.07 & 0.1 \\
\hline & 1.08 & 0.13 & 2.28 & 0.04 & 2.32 & 0.38 & 2.26 & 0.07 & 0.91 & 0.11 & 0.58 & 0.10 & 0.15 & 0.03 & 0.10 & 0.07 & 0.05 \\
\hline \multirow{2}{*}{$\stackrel{0}{+}$} & 1.09 & 0.09 & 3.82 & 0.07 & 2.53 & 0.12 & 1.46 & 0.11 & 0.22 & 0.05 & 0.31 & 0.04 & 0.28 & 0.04 & 0.11 & 0.06 & 0.1 \\
\hline & 1.88 & 0.04 & 2.08 & 0.07 & 0.82 & 0.12 & 0.81 & 0.08 & 0.18 & 0.05 & 0.22 & 0.03 & 0.17 & 0.04 & 0.10 & 0.06 & 0.05 \\
\hline & & & & & & & & & & & 33 & & & & & & \\
\hline & \multicolumn{16}{|c|}{ Number of Measurements (Measurement Frequency [Sol-1 ${ }^{-1}$ ) } & \\
\hline
\end{tabular}

\section{Acknowledgements}

The authors would like to acknowledge the helpful critical reviews of Dr Matthias Grott of the Institut für Planetenforschung, Deutsches Zentrum für Luft- und Raumfahrt e.V. (DLR), in Berlin. The research behind the presented results is supported by grants from DLR and The Open University.

\section{References}

BANASZKIEWICZ, M., SEIFERLIN, K., SPOHN, T., KARGL, G. \& KÖMLE, N. 1997. A new method for the determination of thermal conductivity and thermal diffusivity from linear heat source measurements. Review of Scientific Instruments, 68, 4184-4190.

BANERDT, W. B., SMREKAR, S., ALKALAI, L., HOFFMAN, T., WARWICK, R., HURST, K., FOLKNER, W., LOGNONNÉ, P., SPOHN, T., ASMAR, S., BANFIELD, D., BOSCHI, L., 
CHRISTENSEN, U., DEHANT, V., GIARDINI, D., GOETZ, W., GOLOMBEK, M., GROTT, M., HUDSON, T., JOHNSON, C., KARGL, G., KOBAYASHI, N., MAKI, J., MIMOUN, D., MOCQUET, A., MORGAN, P., PANNING, M., PIKE, W. T., TROMP, J., VAN ZOEST, T., WEBER, R., WIECZOREK, M. \& TEAM, I. 2012. InSight: An Integrated Exploration of the Interior of Mars. In: Lunar and Planetary Institute Science Conference Abstracts, March 1, 2012 2012. 43, 2838.

BOEHLER, R. 1996. Melting Temperature of the Earth's Mantle and Core: Earth's Thermal Structure. Annual Review of Earth And Planetary Sciences, 24, 15-40.

BOYNTON, W. V., FELDMAN, W. C., SQUYRES, S. W., PRETTYMAN, T. H., BRÜCKNER, J., EVANS, L. G., REEDY, R. C., STARR, R., ARNOLD, J. R., DRAKE, D. M., ENGLERT, P. A. J., METZGER, A. E., MITROFANOV, I., TROMBKA, J. I., D'USTON, C., WÄNKE, H., GASNAULT, O., hAMARA, D. K., JANES, D. M., MARCIALIS, R. L., MAURICE, S., MIKHEEVA, I., TAYLOR, G. J., TOKAR, R. \& SHINOHARA, C. 2002. Distribution of Hydrogen in the Near Surface of Mars: Evidence for Subsurface Ice Deposits. Science, 297, 81-85.

BREUER, D. \& SPOHN, T. 2003. Early plate tectonics versus single-plate tectonics on Mars: Evidence from magnetic field history and crust evolution. Journal of Geophysical Research (Planets), $108,5072$.

CHRISTENSEN, P. R., BANDFIELD, J. L., HAMILTON, V. E., RUFF, S. W., KIEFFER, H. H., TITUS, T. N., MALIN, M. C., MORRIS, R. V., LANE, M. D., CLARK, R. L., JAKOSKY, B. M., MELLON, M. T., PEARL, J. C., CONRATH, B. J., SMITH, M. D., CLANCY, R. T., KUZMIN, R. O., ROUSH, T., MEHALL, G. L., GORELICK, N., BENDER, K., MURRAY, K., DASON, S., GREENE, E., SILVERMAN, S. \& GREENFIELD, M. 2001. Mars Global Surveyor Thermal Emission Spectrometer experiment: Investigation description and surface science results. Journal of Geophysical Research, 106, 2382323872.

DEHANT, V., BANERDT, B., LOGNONNÉ, P., GROTT, M., ASMAR, S., BIELE, J., BREUER, D., FORGET, F., JAUMANN, R., JOHNSON, C., KNAPMEYER, M., LANGLAIS, B., LE FEUVRE, M., MIMOUN, D., MOCQUET, A., READ, P., RIVOLDINI, A., ROMBERG, O., SCHUBERT, G., SMREKAR, S., SPOHN, T., TORTORA, P., ULAMEC, S. \& VENNERSTRØM, S. 2012. Future Mars geophysical observatories for understanding its internal structure, rotation, and evolution. Planetary and Space Science, 68, 123-145.

GOLOMBEK, M., WARNER, N., SCHWARTZ, C. \& GREEN, J. 2013. Surface Characteristics of Prospective InSight Landing Sites in Elysium Planitia. In: Lunar and Planetary Science Conference, March 1, 2013 2013. 44, 1696.

GOLOMBEK, M., WIGTON, N., BLOOM, C., SCHWARTZ, C., KANNAN, S., KIPP, D., HUERTAS, A. \& BANERDT, B. 2014. Final Four Landing Sites for the InSight Geophysical Lander. In: Lunar and Planetary Science Conference, March 1, 2014 2014. 45, 1499.

GROTT, M. \& BREUER, D. A. A. 2010. On the spatial variability of the Martian elastic lithosphere thickness: Evidence for mantle plumes? Journal of Geophysical Research (Planets), 115, 3005. 
GROTT, M., HELBERT, J. \& NADALINI, R. 2007. Thermal structure of Martian soil and the measurability of the planetary heat flow. J. Geophys. Res., 112, E09004.

HAGERMANN, A. 2005. Planetary heat flow measurements. Royal Society of London Transactions Series A, vol. 363, 2777-2791.

HAGERMANN, A. \& TANAKA, S. 2006. Ejecta deposit thickness, heat flow, and a critical ambiguity on the Moon. Geophysical Research Letters, 33, L19203.

HAUCK, S. A. \& PHILLIPS, R. J. 2002. Thermal and crustal evolution of Mars. Journal of Geophysical Research (Planets), 107, 5052.

HEIKEN, G. H., VANIMAN, D. T., FRENCH, B. M., VANIMAN, D. T. \& FRENCH, B. M. 1991. Lunar sourcebook - A user's guide to the moon.

JUSTH, H. L., JUSTUS, C. G., RAMEY, H. S. \& MILLOUR, E. 2011. Mars-Gram 2010: Improving the precison of Mars-Gram. In: FORGET, F., ed. Mars Atmosphere: Modelling and observation, February 1, 2011 2011. 265-267.

KIEFFER, H. H., MARTIN, T. Z., PETERFREUND, A. R., JAKOSKY, B. M., MINER, E. D. \& PALLUCONI, F. D. 1977. Thermal and albedo mapping of Mars during the Viking primary mission. Journal of Geophysical Research, 82, 4249-4291.

LANGSETH, M. G., JR., CLARK, S. P., JR., CHUTE, J. L., JR., KEIHM, S. J. \& WECHSLER, A. E. 1972. The Apollo 15 Lunar Heat-Flow Measurement. Moon, 4, 390-410.

LANGSETH, M. G., KEIHM, S. J. \& PETERS, K. 1976. Revised lunar heat-flow values. In: KINSLER, D. C., ed. Lunar and Planetary Science Conference Proceedings, April 1, 1976 1976. 7, 3143-3171.

MARS CLIMATE DATABASE. 2013. Martian Seasons and Solar Longitude [Online]. Available: http://www-mars.Imd.jussieu.fr/mars/time/solar_longitude.html [Accessed 13/09/2013 2013].

MELLON, M. T., JAKOSKY, B. M., KIEFFER, H. H. \& CHRISTENSEN, P. R. 2000. High-Resolution Thermal Inertia Mapping from the Mars Global Surveyor Thermal Emission Spectrometer. Icarus, 148, 437-455.

MILlOUR, E., SPIGA, A., COLAITIS, A., NAVARRO, T., MADELEINE, J.-B., CHAUFFRAY, J.-Y., MONTABONE, L., LOPEZ-VALVERDE, M. A., GONZALEZ-GALINDO, F., LEFÈVRE, F., MONTMESSIN, F., LEWIS, S. R., READ, L. P., DESJEAN, M.-C. \& HUOT, J.-P. 2012. Mars Climate Database Version 5. In: European Planetary Science Congress 2012, September 1, 2012 2012. 302.

NASA, A. R. C. D. T. 2014. NASA App. Missions. 1.43 ed. Google Play Store: National Aeronautics and Space Administration.

NOWICKI, S. A. \& CHRISTENSEN, P. R. 2007. Rock abundance on Mars from the Thermal Emission Spectrometer. Journal of Geophysical Research (Planets), 112, 5007. 
PIQUEUX, S. \& CHRISTENSEN, P. R. 2009a. A model of thermal conductivity for planetary soils: 1. Theory for unconsolidated soils. Journal of Geophysical Research (Planets), 114, 9005.

PIQUEUX, S. \& CHRISTENSEN, P. R. 2009b. A model of thermal conductivity for planetary soils: 2 . Theory for cemented soils. Journal of Geophysical Research (Planets), 114, 9006.

PIQUEUX, S. \& CHRISTENSEN, P. R. 2011. Temperature-dependent thermal inertia of homogeneous Martian regolith. Journal of Geophysical Research (Planets), 116, 7004.

POLLACK, H. N., HURTER, S. J. \& JOHNSON, J. R. 1993. Heat flow from the earth's interior Analysis of the global data set. Reviews of Geophysics, 31, 267-280.

ROBIE, R. A., HEMINGWAY, B. S. \& WILSON, W. H. 1970. Specific heats of lunar surface materials from $90^{\circ}$ to $350^{\circ} \mathrm{K}$. In: LEVINSON, A. A., ed. Lunar and Planetary Science Conference Proceedings, March 4 1970. 3, 2361-2367.

RUDNICK, R. L. \& FOUNTAIN, D. M. 1995. Nature and composition of the continental crust: A lower crustal perspective. Reviews of Geophysics, 33, 267-310.

RUFF, S. W. \& CHRISTENSEN, P. R. 2002. Bright and dark regions on Mars: Particle size and mineralogical characteristics based on Thermal Emission Spectrometer data. Journal of Geophysical Research (Planets), 107, 5127.

SEIFERLIN, K., KÖMLE, N. I., KARGL, G. \& SPOHN, T. 1996. Line heat-source measurements of the thermal conductivity of porous $\mathrm{H}_{2} \mathrm{O}$ ice, $\mathrm{CO}_{2}$ ice and mineral powders under space conditions. Planetary and Space Science, 44, 691-704.

SHEN, P. Y. \& BECK, A. E. 1991. Least Squares Inversion of Borehole Temperature Measurements in Functional Space. J. Geophys. Res., 96, 19965-19979.

SHEN, P. Y. \& BECK, A. E. 1992. Paleoclimate change and heat flow density inferred from temperature data in the Superior Province of the Canadian sheild. Palaeogeography, Palaeoclimatology, Palaeoecology, 98, 143-165.

SPOHN, T., BALL, A. J., SEIFERLIN, K., CONZELMANN, V., HAGERMANN, A., ÖMLE, N. I. \& KARGL, G. 2001. A heat flow and physical properties package for the surface of Mercury. Planetary and Space Science, 49, 1571-1577.

SPOHN, T., GROTT, M., KNOLLENBERG, J., VAN ZOEST, T., KARGL, G., SMREKAR, S. E., BANERDT, W. B., HUDSON, T. L. \& TEAM, H. I. 2012. INSIGHT: Measuring the Martian Heat Flow Using the Heat Flow and Physical Properties Package $\left(\mathrm{HP}^{3}\right)$. In: Lunar and Planetary Institute Science Conference, 2012. 43, 1445.

StACK, K. M., GROTZINGER, J. P., SUMNER, D. Y., EHLMANN, B. L., MILLIKEN, R. E., EIGENBRODE, J. L., GUPTA, S., WILLIAMS, R. M. E., KAH, L. C., LEWIS, K. W. \& TEAM, M. S. 2013. Using Outcrop Exposures on the Road to Yellowknife Bay to Build a Stratigraphic Column, Gale 
Crater, Mars. In: Lunar and Planetary Institute Science Conference Abstracts, March 1 2013. 44, 1431.

TARANTOLA, A. 2005. Inverse problem theory and methods for model parameter estimation, Philadelphia, PA, Society for Industrial and Applied Mathematics.

TARANTOLA, A. \& VALETTE, B. 1982. Generalized nonlinear inverse problems solved using the least squares criterion. Reviews of Geophysics, 20, 219-232.

WANG, K. 1992. Estimation of Ground Surface Temperatures from Borehole Temperature Data. J. Geophys. Res., 97, 2095-2106.

WARNER, N. H., GOLOMBEK, M. P., BLOOM, C., WIGTON, N. \& SCHWARTZ, C. 2014. Regolith Thickness in Western Elysium Planitia: Constraints for the InSight Mission. In: Lunar and Planetary Science Conference, March 1, 2014 2014. 45, 2217.

WARREN, P. H. \& RASMUSSEN, K. L. 1987. Megaregolith insulation, internal temperatures, and bulk uranium content of the moon. Journal of Geophysical Research, 92, 3453-3465.

WIGTON, N. R., WARNER, N. \& GOLOMBEK, M. 2014. Terrain Mapping of the InSight Landing Region: Western Elysium Planitia, Mars. In: Lunar and Planetary Science Conference, March 1, 2014 2014. 45, 1234. 